\title{
Hadroquarkonium from lattice QCD
}

\author{
Maurizio Alberti, ${ }^{1}$ Gunnar S. Bali, ${ }^{2,3}$ Sara Collins, ${ }^{2}$ Francesco Knechtli, ${ }^{1}$ Graham Moir, ${ }^{4}$ and Wolfgang Söldner ${ }^{2}$ \\ ${ }^{1}$ Department of Physics, Bergische Universität Wuppertal, Gaußstraße 20, 42119 Wuppertal, Germany \\ ${ }^{2}$ Institut für Theoretische Physik, Universität Regensburg, \\ Universitätsstraße 31, 93053 Regensburg, Germany \\ ${ }^{3}$ Department of Theoretical Physics, Tata Institute of Fundamental Research, \\ Homi Bhabha Road, Mumbai 400005, India \\ ${ }^{4}$ Department of Applied Mathematics and Theoretical Physics, Centre for Mathematical Sciences, \\ University of Cambridge, Wilberforce Road, Cambridge CB3 OWA, United Kingdom
}

(Received 24 August 2016; revised manuscript received 27 February 2017; published 3 April 2017)

\begin{abstract}
The hadroquarkonium picture [S. Dubynskiy and M.B. Voloshin, Phys. Lett. B 666, 344 (2008)] provides one possible interpretation for the pentaquark candidates with hidden charm, recently reported by the LHCb Collaboration, as well as for some of the charmoniumlike " $X, Y, Z$ ' states. In this picture, a heavy quarkonium core resides within a light hadron giving rise to four- or five-quark/antiquark bound states. We test this scenario in the heavy quark limit by investigating the modification of the potential between a static quark-antiquark pair induced by the presence of a hadron. Our lattice QCD simulations are performed on a Coordinated Lattice Simulations (CLS) ensemble with $N_{f}=2+1$ flavors of nonperturbatively improved Wilson quarks at a pion mass of about $223 \mathrm{MeV}$ and a lattice spacing of about $a=0.0854 \mathrm{fm}$. We study the static potential in the presence of a variety of light mesons as well as of octet and decuplet baryons. In all these cases, the resulting configurations are favored energetically. The associated binding energies between the quarkonium in the heavy quark limit and the light hadron are found to be smaller than a few MeV, similar in strength to deuterium binding. It needs to be seen if the small attraction survives in the infinite volume limit and supports bound states or resonances.
\end{abstract}

DOI: 10.1103/PhysRevD.95.074501

\section{INTRODUCTION}

Recently, the LHCb Collaboration found two structures in the decay $\Lambda_{b} \rightarrow J / \psi p K$, which can be interpreted as candidates for pentaquark states with hidden charm, containing three light quarks, in addition to a charm quark-antiquark pair $[1,2]$. The most likely spin and parity assignments for these candidates, labeled $P_{c}^{+}(4380)$ and $P_{c}^{+}(4450)$, are $J^{P}=3 / 2^{-}$and $5 / 2^{+}$, respectively, with $3 / 2^{+}$and $5 / 2^{-}$being another possibility. While the nature of these (and of some other structures) is still disputed [3,4], the number of established charmonium resonances certainly has exploded during the past 15 years, see Ref. [5] and, e.g., Ref. [6] for a more recent review. Many of these are of an exotic nature and some clearly hint at light quark-antiquark or-in the case of the $P_{c}$ candidates-even at $q q q$ components, in addition to the charm quark and antiquark.

Many models can accommodate some, or if extended to include states that contain five (anti-)quarks, even all of these resonances: tetraquarks [7-9] consisting of diquark-antidiquark pairs, including a recently proposed "dynamic" picture [10,11], molecules of two open charm mesons [12-16], hybrid states [17-20] containing a charm quark-antiquark pair and additional valence gluons, hadrocharmonium with a compact charmonium core bound inside a light hadron [21,22], and mixtures of the above. Here we will specifically aim to establish if the last picture (hadroquarkonium) is supported in the heavy quark limit.
The standard way of addressing a strongly decaying resonance and extracting the position of the associated pole in the unphysical Riemann sheet from simulations in Euclidean spacetime boxes was introduced by Lüscher [23]. For applications of this and related methods to charmonium spectroscopy, see, e.g., Ref. [24] and references therein. In the case of charmonia, this is particularly challenging since, in addition to ground states, radial excitations need to be considered and the number of different decay channels can be large, some with more than two hadrons in the final state. Moreover, while in principle resonance parameters can be computed, at least below inelastic multiparticle thresholds, these will not necessarily tell us much about the "nature" of the underlying state: how does the naive quark model need to be modified to provide a guiding principle for the existence or nonexistence of an exotic resonance?

A direct computation of the scattering parameters of, e.g., a nucleon-charmonium resonance in a realistic setting constitutes a serious computational challenge, especially if one aims at conclusive results with meaningful errors. Instead of directly approaching the problem at hand, here we restrict ourselves to the heavy quark limit in which the charm quarks can be considered as slowly moving in the background of gluons, sea quarks and, possibly, light hadrons.

After integrating out the degrees of freedom associated with the heavy quark mass $m_{Q}$, quarkonia can be described 
in terms of an effective field theory: nonrelativistic QCD (NRQCD) [25]. In the limit of small distances $r$, or equivalently, large momentum transfers $m_{Q} v$, where $v$ is the interquark velocity, the scale $m_{Q} v \sim 1 / r$ can also be integrated out, resulting in potential NRQCD (pNRQCD) $[26,27]$. Then, to leading order in $r$ with respect to the pNRQCD multipole expansion and to $v^{2} \sim \alpha_{s}$ in the NRQCD power counting, quarkonium becomes equivalent to a nonrelativistic quantum mechanical system, where the interaction potential is given by the static potential $V_{0}(r)$ which can, e.g., be computed nonperturbatively from Wilson loop expectation values $\langle W(r, t)\rangle$ in Euclidean spacetime,

$$
V_{0}(r)=-\lim _{t \rightarrow \infty} \frac{\mathrm{d}}{\mathrm{d} t} \ln \langle W(r, t)\rangle .
$$

Here we investigate whether this potential becomes modified in the presence of a light hadron. This would then lower or increase quarkonium energy levels. If embedding the quarkonium in the light hadron is energetically favorable, this would suggest a bound state, at least for sufficiently large quark masses.

This article is organized as follows. In Sec. II we briefly discuss previous studies of nucleon-charmonium bound states and comment on the ordering of scales that we consider. In Sec. III we define the observables that we compute. Then, in Sec. IV we describe details of the simulation, before numerical results are presented in Sec. V. Subsequently, in Sec. VI we relate the modifications of the static potential to quarkonium bound state energies, before we summarize in Sec. VII.

\section{NUCLEON-CHARMONIUM BOUND STATES}

Light meson exchanges between a single nucleon or nucleons bound in a nucleus and quarkonium, which does not contain any light valence quarks, are suppressed by the Zweig rule. Therefore, such interactions should be dominated by gluon exchanges. In the heavy quark limit, quarkonium can be considered essentially as a point particle of a heavy quark and antiquark bound by the short-range perturbative Coulomb potential. The first nonvanishing chromodynamical multipole is then a dipole and quarkonium may interact with the nuclear environment via color dipole-dipole van der Waals forces. For a recent discussion of the relevant scales in the context of effective field theories, see Ref. [28]. Initially, using phenomenological interaction potentials, nucleon-charmonium binding energies ranging from $20 \mathrm{MeV}$ [29,30] down to $10 \mathrm{MeV}$ [31] were estimated for nuclei consisting of $A>3[29,31]$ and $A>10$ [30] nucleons. A first QCD-based estimate [32] for the potential between quarkonium in the heavy quark limit and a nucleus resulted in $\Upsilon$ and $J / \psi$ binding energies of a few $\mathrm{MeV}$ and $10 \mathrm{MeV}$, respectively, possibly with large relativistic and higher-order multipole corrections in the charmonium case. This discussion of light nuclei hosting a quarkonium state may have contributed to the suggestion of quarkonium states that are embedded within light hadrons, hadroquarkonia [22].

At present no (p)NRQCD lattice studies of baryoncharmonium states exist. However, a few investigations employing relativistic charm quarks have been carried out. In Ref. [33], the $\eta_{c}$ and $J / \psi$ charmonia were scattered with light pseudoscalar and vector mesons as well as with the nucleon, in the quenched approximation with rather large light quark mass values; the ratio $M_{\pi} / m_{\rho}$ ranged from 0.9 down to 0.68 . Varying the lattice extent from $L=1.6 \mathrm{fm}$ over $2.2 \mathrm{fm}$ up to $3.2 \mathrm{fm}$, in this pioneering work scattering lengths were extracted, indicating some attraction in all the channels investigated. A similar study was performed in Ref. [34], combining staggered sea with domain wall light and Fermilab charm quarks; however, unusually small scattering lengths were reported. Finally, a pseudoscalar charm quark-antiquark pair was created along with a nucleon and even with light nuclei by the NPLQCD Collaboration [35]. In this work the binding energy reported for the nucleon case was about $20 \mathrm{MeV}$, albeit at a rather large light quark mass value, corresponding to $M_{\pi} \approx 800 \mathrm{MeV}$, and for a coarse lattice spacing $a \approx 0.145 \mathrm{fm}$. This value of the binding energy is consistent with some of the expectations for charmonia in a nuclear environment discussed above.

Closest in spirit to the van der Waals interaction picture, Kawanai and Sasaki [36] in a quenched study, again at rather large pion masses, $M_{\pi} \geq 640 \mathrm{MeV}$, computed a charmonium-nucleon Bethe-Salpeter wave function. Plugging this into a Schrödinger equation, a potential between the charmonium and the nucleon was extracted, indicating very weak attractive forces.

Here we will not assume a nonrelativistic light hadron of mass $m_{H}$, whose dipole-dipole interaction with quarkonium can be described by a potential. Instead, our light hadron is an extended relativistic object. We also go beyond the pointdipole approximation in the heavy quark sector by "pulling" quark and antiquark apart by a distance $r$. We then determine the modification of the interaction potential between the heavy quark-antiquark pair, that we approximate as static sources, induced by the presence of a light hadron. To be more precise, we will consider the limit $m_{Q} \gg m_{H}$, $m_{Q} \gg \Lambda_{\mathrm{QCD}}$, where $\Lambda_{\mathrm{QCD}}$ denotes a typical nonperturbative scale of a few hundred $\mathrm{MeV}$, and $v^{2} \ll 1$. Since we determine the quark-antiquark potential, i.e., the matching function between NRQCD and pNRQCD, nonperturbatively, $m_{Q} v \sim$ $1 / r$ does not need to be much larger than $\Lambda_{\mathrm{QCD}}$. However, we neglect color octet contributions [26,27], which may become significant at distances $r \gtrsim \Lambda_{\mathrm{QCD}}^{-1}$.

\section{STATIC POTENTIALS “INSIDE” HADRONS}

We denote an interpolator creating a static fundamental color charge $Q$ at a position $\mathbf{z}+\mathbf{r} / 2$ and destroying it at a position $\mathbf{z}-\mathbf{r} / 2$ as $\mathcal{Q}_{\mathbf{r}}^{\dagger}(\mathbf{z})$. This will transform according to 
HADROQUARKONIUM FROM LATTICE QCD

the fundamental 3 representation of the gauge group at $\mathbf{z}+$ $\mathbf{r} / 2$ and according to $3^{*}$ at $\mathbf{z}-\mathbf{r} / 2$ and hence it contains a gauge covariant transporter connecting these two points (usually a spatially smeared Schwinger line). The Wilson loop can then be written as

$$
\langle W(r, t)\rangle=\left\langle 0\left|\mathcal{Q}_{r} \mathcal{T}^{t / a} \mathcal{Q}_{r}^{\dagger}\right| 0\right\rangle,
$$

where we assume rotational invariance is restored for $r=|\mathbf{r}| \gg a$, and $\mathcal{T}=e^{-a \Vdash}$ denotes the transfer matrix connecting adjacent time slices.

Within the static approximation, there are different strategies to investigate bound states containing a heavy quark-antiquark pair and additional light quarks. One method, which we are not going to pursue here, amounts to creating a light hadron $H$ containing either $\bar{q} q$ or $q q q$ along with the stringy $Q \bar{Q}$ state at equal Euclidean time. The interpolator for creating a zero-momentum projected tetra- or pentaquark state then has the form

$$
\overline{\mathcal{P}}_{r}=\sum_{\mathbf{z}} \overline{\mathcal{H}}(\mathbf{z}) \mathcal{Q}_{r}^{\dagger}(\mathbf{z})
$$

Note that the creation interpolator $\overline{\mathcal{H}}$ of a hadronic state (as well as $\overline{\mathcal{P}}_{r}$ ) will carry a spinor index, which we suppress. The correlator of interest is now $\left\langle 0\left|\mathcal{P}_{r} \mathcal{T}^{t / a} \overline{\mathcal{P}}_{r}\right| 0\right\rangle$. Even without summing over positions $\mathbf{z}$ this is automatically projected onto zero momentum at source and sink as the light hadron is tied in position space to the static quarks, see Eq. (3). Numerous possibilities exist for where to spatially place the light quarks relative to the heavy sources within the interpolator $\mathcal{P}_{r}$ and how to transport and contract the color such that the interpolator respects the correct gauge transformation properties. This freedom can be exploited to enhance the overlap of $\overline{\mathcal{P}}_{r}|0\rangle$ with the physical state and may also provide some insight into its internal structure.

Subsequent to a pioneering lattice study [37] of a light $q q$ pair bound in the above way to two static antitriplet sources, quite a few simulations of a light $q \bar{q}$ pair bound to the string state created by $\mathcal{Q}_{r}^{\dagger}$ have also been carried out. Such results exist both for a light quark-antiquark pair with isospin $I=1[38-41]$ and $I=0[39,42]$. In contrast, a static quark-antiquark pair accompanied by three light quarks has not been investigated on the lattice so far.

Instead of creating tetra- or pentaquark states containing a heavy or static quark and the corresponding antiquark, here we wish to "directly" address a particular picture of such bound states, hadroquarkonium [21,22]. This will be achieved by computing differences between the static potential in the presence of a light hadron, relative to the static potential in the vacuum. The former can be obtained from the large Euclidean time decay of

$$
\left\langle H\left|\mathcal{Q}_{r} \mathcal{T}^{t / a} \mathcal{Q}_{r}^{\dagger}\right| H\right\rangle
$$

PHYSICAL REVIEW D 95, 074501 (2017)

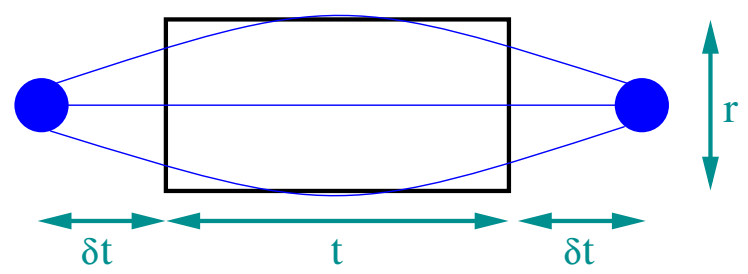

FIG. 1. Graphical representation of the four-point correlation function in the numerator of Eq. (6) for the example of a static quark-antiquark pair at a distance $r$ embedded in a baryon. Thin blue lines correspond to light quark propagators and the black rectangle to the Wilson loop.

where $|H\rangle$ is the ground state that is destroyed by the zeromomentum interpolator

$$
\mathcal{H} \equiv \sum_{\mathbf{x}} \mathcal{H}(\mathbf{x})
$$

In order to evaluate the expectation value Eq. (4) we create a hadronic state at time 0 . We then let it propagate to $\delta t$ to achieve ground-state dominance. At this time we create an additional quark-antiquark string by inserting a (smeared) Wilson loop of time extent $t$, which terminates at $t+\delta t$. Finally, we destroy the light hadron at the time $t+2 \delta t$. Then

$$
\left\langle H\left|\mathcal{Q}_{r} \mathcal{T}^{t / a} \mathcal{Q}_{r}^{\dagger}\right| H\right\rangle \propto \lim _{\delta t \rightarrow \infty} \frac{\left\langle 0\left|\mathcal{H} \mathcal{T}^{\delta t / a} \mathcal{Q}_{r} \mathcal{T}^{t / a} \mathcal{Q}_{r}^{\dagger} \mathcal{T}^{\delta t / a} \overline{\mathcal{H}}\right| 0\right\rangle}{\left\langle 0\left|\mathcal{H} \mathcal{T}^{(t+2 \delta t) / a} \overline{\mathcal{H}}\right| 0\right\rangle}
$$

where we average over all spatial Wilson loop positions $\mathbf{z}$ and light hadronic sink positions $\mathbf{x}$. Zero-momentum projection at the light hadronic source can be avoided, due to the translational invariance of expectation values. The correlator of interest is depicted in Fig. 1.

We can now define the potential in the background of the hadron as

$$
V_{H}(r)=-\lim _{t \rightarrow \infty} \frac{\mathrm{d}}{\mathrm{d} t} \ln \left\langle H\left|\mathcal{Q}_{r} \mathcal{T}^{t / a} \mathcal{Q}_{r}^{\dagger}\right| H\right\rangle,
$$

in analogy to Eqs. (1) and (2). In the end we will compute differences

$$
\begin{aligned}
\Delta V_{H}(r) & =V_{H}(r)-V_{0}(r) \\
& =-\lim _{t \rightarrow \infty} \frac{\mathrm{d}}{\mathrm{d} t} \ln \frac{\left\langle H\left|\mathcal{Q}_{r} \mathcal{T}^{t / a} \mathcal{Q}_{r}^{\dagger}\right| H\right\rangle}{\left\langle 0\left|\mathcal{Q}_{r} \mathcal{T}^{t / a} \mathcal{Q}_{r}^{\dagger}\right| 0\right\rangle} \\
& =-\lim _{t \rightarrow \infty} \frac{\mathrm{d}}{\mathrm{d} t} \ln \frac{\left\langle W(r, t) C_{H, 2 \mathrm{pt}}(t+2 \delta t)\right\rangle}{\langle W(r, t)\rangle\left\langle C_{H, 2 \mathrm{pt}}(t+2 \delta t)\right\rangle},
\end{aligned}
$$

where the argument of the logarithm is simply the correlator of a light hadronic two-point function with the Wilson loop inserted, divided by the Wilson loop expectation value 
times the hadronic two-point function $\left\langle C_{H, 2 \mathrm{pt}}(t+2 \delta t)\right\rangle=$ $\left\langle 0\left|\mathcal{H} \mathcal{T}^{(t+2 \delta t) / a} \overline{\mathcal{H}}\right| 0\right\rangle$, see the denominator of Eq. (6).

We are now in the position to address the question within what hadronic channels $\Delta V_{H}(r)$ will be attractive and in what cases repulsive. This may serve as an indicator for the stability of related hadroquarkonia. In view of the recent LHCb result [1,2] baryonic states $|H\rangle$ are particularly interesting. For instance, adding the mass of the $\Delta$ to that of the $J / \psi$ gives $4329 \mathrm{MeV}$ [43], which is not far away from the mass of the $P_{c}(4380)$. Furthermore, $J^{P}=3 / 2^{+}$ can couple to $1^{-}$to give $3 / 2^{-}$. Another example is the sum of the nucleon $(N)$ and $\chi_{c 2}$ masses, $4496 \mathrm{MeV}$, which is close to the mass of the $P_{c}^{+}$(4450). Again, $1 / 2^{+}$and $2^{+}$can couple to $J^{P}=5 / 2^{+}$.

\section{IMPLEMENTATION AND TECHNICAL DETAILS}

We analyse the $N_{f}=2+1$ ensemble "C101", which has a volume of $96 \times 48^{3}$ sites and was generated by the Coordinated Lattice Simulations (CLS) effort [44] using the openQCD simulation program $[45,46]$. Open boundary conditions in time and nonperturbatively order- $a$-improved Wilson fermions on top of the tree-level Symanzik improved Wilson gauge action are employed, see Ref. [44] for details on the simulation. To determine the lattice spacing we extrapolate the scale parameter $t_{0}$ [47] to the physical point, where we obtain $\sqrt{8 t_{0}} / a=4.852$ (7) [48]. Using the continuum limit result $\sqrt{8 t_{0}}=0.4144(59)(37) \mathrm{fm}$ [49] gives $a=0.0854(15) \mathrm{fm}$. The pion and kaon masses on this ensemble are $M_{\pi} \approx 223 \mathrm{MeV}$ and $M_{K} \approx 476 \mathrm{MeV}$, respectively. Note that while the pion is heavier than in nature the kaon is somewhat lighter since the sum of quark masses $2 m_{\ell}+m_{s}\left(m_{\ell}=m_{u}=m_{d}\right)$ was adjusted to a value close to the physical one and kept constant within the main set of CLS simulations [44]. The spatial lattice extent reads $L \approx 4.6 / M_{\pi} \approx 4.1 \mathrm{fm}$. For details see Ref. [48].

We analyse 1552 configurations, separated by four molecular dynamic units. On each of these configurations we place hadronic sources on 12 different time slices $(30,43,44, \ldots, 52,53,65)$ at random spatial positions to reduce autocorrelations. Due to the use of open boundary conditions, we have to discard the boundary regions from our analysis. After carefully checking for translational invariance in time, we use forward and backward propagating hadronic two-point functions for the 11 sources $^{1}$ placed in the central region of the lattice but propagate only forward from $t_{0} / a=30$ and backward from $\left(t-t_{0}\right) / a=65$. This gives a total of $24 \times 1552$ two-point functions for each light hadron and spin polarization considered. Since $\delta t$ needs to be kept small to obtain statistically meaningful results, the quark propagators entering these two-point functions are

\footnotetext{
${ }^{1}$ On time slice 47 two different spatial source positions were used.
}

Wuppertal smeared at source and sink, using spatially smeared gauge transporters, to improve the overlap with the physical ground states.

We measure the Wilson loops using the publicly available WLOOP package [50], following the method described in Ref. [51]. In a first step, all gauge links are smeared using a single iteration of hypercubic (HYP) blocking [52]. Smearing the temporal links corresponds to a particular discretization choice of the static action and results in an exponential improvement of the signal-to-noise ratio of correlators involving static quarks [53]; HYP links reduce the coefficient of the divergent contribution to the selfenergy of a static quark [39,54-56]. In a second step we construct a variational basis of Wilson loops using four different levels $(0,5,7,12)$ of HYP smearing restricted to the three space dimensions.

To enable the construction of the correlators [Eq. (8)], we separately average the Wilson loops for each direction of $\mathbf{r}$, pointing along one of the three spatial lattice axes, and for different temporal positions. As detailed above, due to the use of open boundary conditions, our hadronic two-point functions $C_{H, 2 \mathrm{pt}}(t)$ are confined to the central time region of the lattice. We checked that ratios of Wilson loop expectation values, averaged over different temporal domains, centered about the middle of the lattice, were statistically consistent with 1. Furthermore, Eq. (8) was evaluated in two ways, restricting the Wilson loop average in the denominator to the same time slices as the averaging performed within the numerator as well as averaging the Wilson loop expectation value in the denominator within the whole region where boundary effects were negligible, from time slice 24 to 72 . The two results obtained for each quantity were statistically compatible with each other and below we will make use of the larger averaging region as this resulted in slightly smaller statistical errors.

For the error analysis, we apply the standard method of Ref. [57]. We include the reweighting factors due to twisted-mass reweighting and the rational approximation for the strange quark, see Ref. [46]. We checked that carrying out a more conservative analysis, estimating the effect of slow modes [58], only affects the errors in very few cases and never by more than $30 \%$.

The distance $\mathbf{r}$ between the static sources breaks the continuum $O(3)$ symmetry down to the cylindrical subgroup $\mathrm{O}(2) \otimes \mathbb{Z}_{2}=D_{\infty h}$. Regarding fermionic representations, i.e., for baryons, the double cover is reduced accordingly. In our implementation the static source-antisource distance $\mathbf{r}$ is kept parallel to lattice axes. This means that the 48 element octahedral crystallographic group with reflections $O_{h}$ is reduced to its 16 element subgroup $D_{4 h}$ (and its double cover $O_{h}^{\prime}$ to $D i h_{4} \otimes D i h_{1}$ ). Therefore, when correlating hadrons with a continuum spin assignment $J \geq 1$ with the string state in the $\Sigma_{g}^{+}$irreducible representation (irrep) of $D_{\infty h}\left(A_{1 g}\right.$ of $D_{4 h}$ on the lattice), care has to be taken to construct the adequate irrep of the 


\section{HADROQUARKONIUM FROM LATTICE QCD}

cylindrical group. Below we address the continuum situation but we have checked that the same arguments hold regarding the lattice irreps that we use. In the case of vector mesons, for example, the $\phi$ meson, the $1^{-} \mathrm{O}(3)$ irrep will split into $\Pi_{u}$ and $\Sigma_{u}^{+}$, the latter also appearing in the pseudoscalar channel. To block out this undesired contribution, we need to correlate a Wilson loop with $\mathbf{r}$ pointing in the $z$ direction with the vector state destroyed by a polarized interpolator $\left(\phi_{x}+i \phi_{y}\right) / \sqrt{2}$. We average over cyclic permutations of $x, y$, and $z$. The decuplet baryon interpolator we use, for example for the $\Delta$ baryon, gives a state maximally polarized in the $z$ direction. This then has to be correlated with a Wilson loop pointing in the $z$ direction too, to guarantee $\Lambda=\left|J_{z}\right|=3 / 2$ and to avoid mixing with spin- $1 / 2$ baryonic states. In this case we only used one polarization and therefore we cannot exploit averaging over different directions.

\section{NUMERICAL RESULTS}

Our strategy for testing the hadroquarkonium picture is to determine the potential between two static quarks in the vacuum and to compare this with its counterpart in the presence of a hadron. An energetically favorable difference may signal a tendency of the system to bind. In Sec. VA we discuss the quality of our light hadronic effective masses and in Sec. V B we determine the potential in the vacuum, before moving on to Sec. VC where we investigate the modifications induced by the presence of hadrons. We delay the discussion of the phenomenological consequences to Sec. VI.

\section{A. Light hadronic effective masses}

In the determination of $\Delta V_{H}(r)$ below we will quote the $\delta t=\delta t_{\mathrm{opt}}=5 a \approx 0.43 \mathrm{fm}$ estimates as our final results. With this $\delta t$ value, the fit in $t$ to the right-hand side of Eq. (8) is dominated by data with $t \leq t_{\max }=10 a$. Therefore, the hadronic effective masses

$$
m_{H, \text { eff }}(t+a / 2) \equiv a^{-1} \ln \frac{C_{H, 2 \mathrm{pt}}(t)}{C_{H, 2 \mathrm{pt}}(t+a)}
$$

should ideally exhibit plateaus for $t \ll t_{\max }+2 \delta t_{\mathrm{opt}}=$ $20 a \approx 1.7 \mathrm{fm}$. We wish to check whether this is the case within the given statistics and for the quark smearing that we employ.

In Fig. 2 we display effective masses for some representative hadrons, namely the $K^{*}$, the nucleon $N$, and the cascades $\Xi$ and $\Xi^{*}$, together with one-exponential fits to the plateau region. This region was determined from the requirement that the contribution of the second exponent of a two-exponential fit to data starting at $t=3 a$ amounted to less than $25 \%$ of the error of the correlation function. Using this criterion, indeed, in almost all the cases the plateau starts at $t<10 a=2 \delta t_{\mathrm{opt}}=\left(t_{\max }+2 \delta t_{\mathrm{opt}}\right) / 2$. One of the
PHYSICAL REVIEW D 95, 074501 (2017)

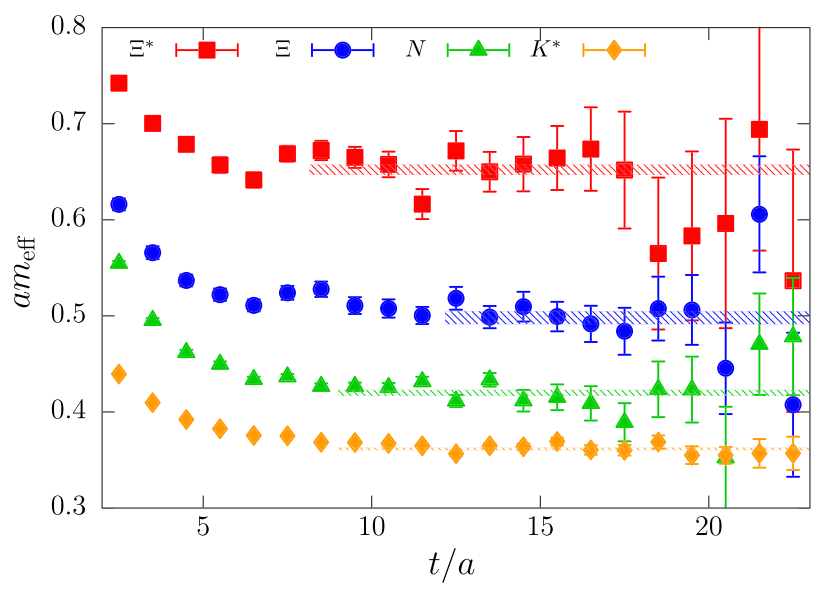

FIG. 2. Effective masses Eq. (9), extracted from various hadronic two-point functions, together with results from oneexponential fits (shaded regions).

few exceptions, that may very well be due to a statistical fluctuation, is the $\Xi$ shown in the figure. We conclude that the ground-state overlap achieved for the light hadrons is sufficient for our purposes.

\section{B. The static potential in the vacuum}

As described in Sec. IV, we determine the static potential, $V_{0}(r)$, from a variational procedure applied to a matrix of correlation functions consisting of spatially smeared Wilson loops. In Fig. 3, we show the physical quantity, $V_{0}(r)-V_{0}\left(\sqrt{8 t_{0}}\right)$, where the subtraction ensures that the self-energies of the static quarks are removed. The value of $V_{0}(r)$ at $r=\sqrt{8 t_{0}}$ was obtained from a local interpolation, cf. Ref. [59]. For later use we also performed a fit to the Cornell parametrization [60]

$$
V_{0}(r)=\mu-\frac{c}{r}+\sigma r
$$

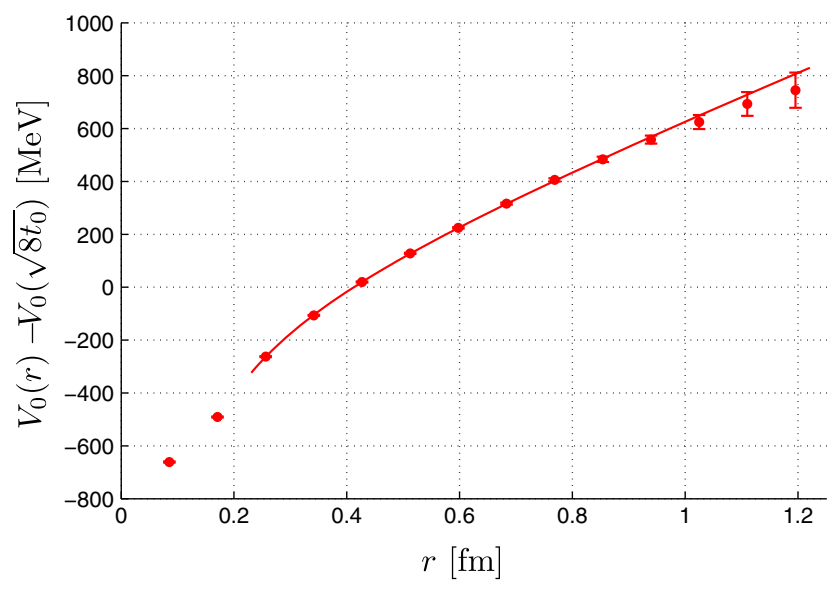

FIG. 3. The quantity $V_{0}(r)-V_{0}\left(\sqrt{8 t_{0}}\right)$, where $V_{0}(r)$ denotes the static quark-antiquark potential in the vacuum, together with the Cornell fit Eq. (10). 
where $\mu$ denotes a constant offset (that diverges in the continuum limit), $\sigma$ is the string tension, and the Coulomb coefficient reads $c=4 \alpha_{s} / 3$ at tree level. The fit with the parameter values,

$$
\begin{aligned}
\mu & =0.721(14) \mathrm{GeV}, \quad c=0.468(14), \\
\sigma & =0.906(16) \mathrm{GeV} / \mathrm{fm},
\end{aligned}
$$

where we used $a=0.0854 \mathrm{fm}$, is also shown in the figure.

To ensure that our results are not tainted by the breaking of the "string" between the static quarks, we only consider the static potential up to $\sim 1.2 \mathrm{fm} \approx 14 a$, the distance for which string breaking is expected to occur [39,61]. At larger distances, the phenomenological parametrization Eq. (10) is no longer valid and additional interpolating operators would be required to extract the true ground state. From the static potential, we compute the static force $F=V^{\prime}(r)$ and determine the Sommer scale [62], $r_{0} \approx 0.5 \mathrm{fm}$, from the equation $\left.r^{2} F(r)\right|_{r=r_{0}}=1.65$, obtaining $r_{0} / a=5.890(41)$. We determine $r_{0}$ from a local interpolation of the static force as it is explained in [51]. Indeed, at our lattice spacing and quark mass values, $r_{0} \approx 5.89 a \approx 5.89 \times 0.0854 \mathrm{fm} \approx 0.50 \mathrm{fm}$.

\section{The static potential within a hadron}

We now determine how the presence of a hadron alters the static potential. As discussed in Sec. III, we compute correlation functions

$$
C_{H}(r, \delta t, t)=\frac{\left\langle W(r, t) C_{H, 2 \mathrm{pt}}(t+2 \delta t)\right\rangle}{\langle W(r, t)\rangle\left\langle C_{H, 2 \mathrm{pt}}(t+2 \delta t)\right\rangle},
$$

where we average over the spatial Wilson loop and hadronic sink positions, for different hadrons $H$. For sufficiently large values of $t$ and for fixed values of $r$ and $\delta t$, we can extract the difference between the static potential in the presence of the hadron, $V_{H}(r, \delta t) \stackrel{\delta t \rightarrow \infty}{\rightarrow} V_{H}(r)$, and the vacuum static potential, $V_{0}(r)$, from the exponential decay of this function in Euclidean time,

$$
\begin{aligned}
\Delta V_{H}(r, \delta t) & \equiv V_{H}(r, \delta t)-V_{0}(r) \\
& =-\lim _{t \rightarrow \infty} \frac{\mathrm{d}}{\mathrm{d} t} \ln \left[C_{H}(r, \delta t, t)\right] .
\end{aligned}
$$

As the clover term that appears within the fermionic action extends one unit in time and we have also applied one level of four-dimensional HYP smearing to the Wilson loop, we only consider $\delta t \geq 2 a$. In practice, we obtain statistically meaningful results for $\delta t \lesssim 8 a$, and in some channels even larger values are possible. Note that within Eq. (12) no variational optimization is performed but we restrict ourselves to our highest level of 12 spatial HYP smearing iterations for the Wilson loops.

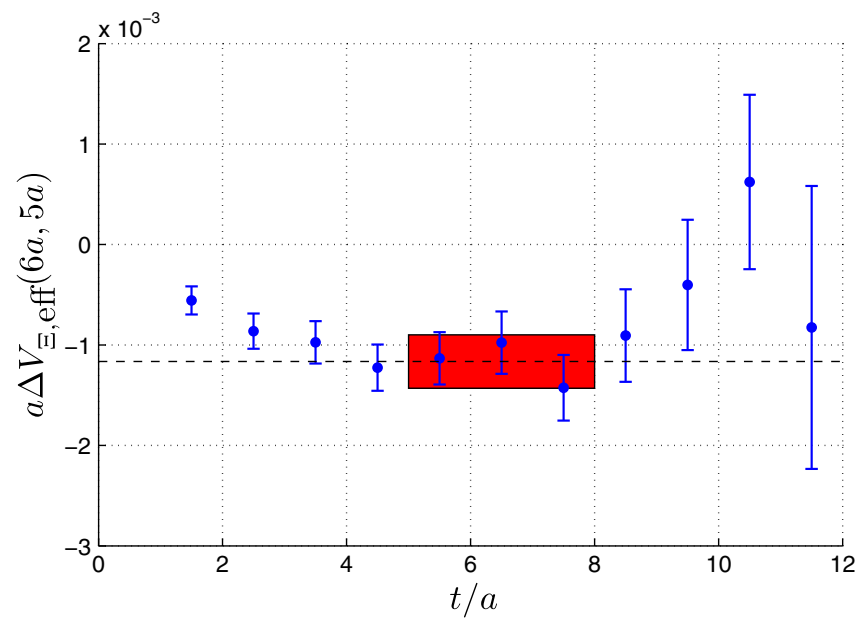

FIG. 4. Effective energy for $\Delta V_{\Xi}(r=6 a, \delta t=5 a)$, defined in Eqs. (12) and (13), as a function of $t$. For the definition of effective energies, see Eq. (9). The error band shows our estimate for $\Delta V(r, \delta t)$, obtained from a linear fit to $\ln C_{H}(r, \delta t, t)$.

For a given hadron and for each combination of $r$ and $\delta t$, we perform linear fits in $t$ to $\ln [C(t, \delta t, t)]$ within the effective energy plateau range. For examples see Figs. 4 and 5 , where we display effective energies for the cascade and the nucleon, respectively, for $r=6 a \approx 0.51 \mathrm{fm}$ and $\delta t=5 a$, together with the results of the corresponding fits. The errors are determined following Ref. [57]. Below we will assign an additional systematic error to our results from varying the fit range.

We will approximate $\Delta V_{H}(r)$ by $\Delta V_{H}(r, \delta t=5 a)$. The functional form is well described by the Cornell parametrization

$$
\Delta V_{H}(r)=\Delta \mu_{H}-\frac{\Delta c_{H}}{r}+\Delta \sigma_{H} r .
$$

The errors on the fit parameters $\Delta \mu_{H}, \Delta c_{H}$, and $\Delta \sigma_{H}$ which we will quote below will be indicative, since they only take

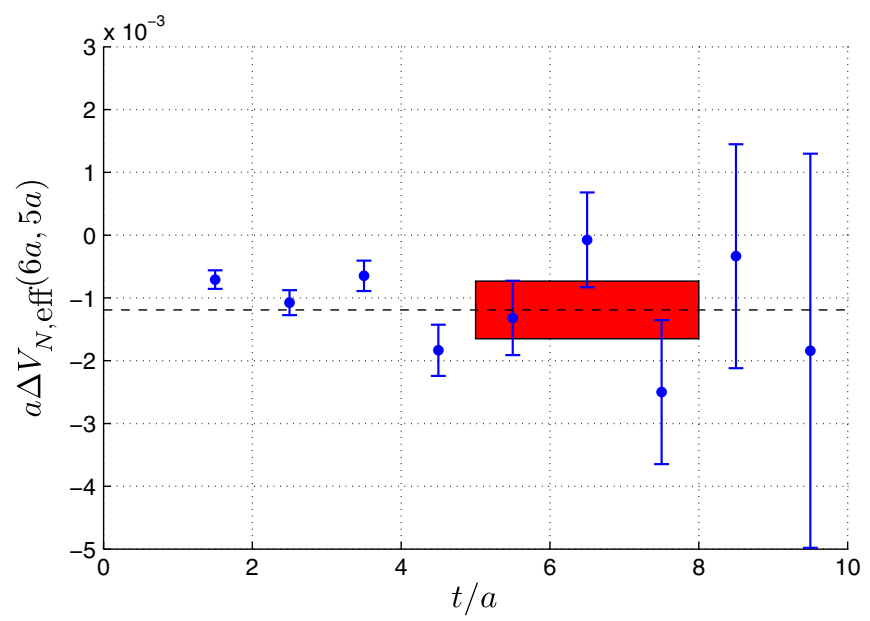

FIG. 5. The same as in Fig. 4 for the nucleon. 
HADROQUARKONIUM FROM LATTICE QCD

into account the statistical errors of $\Delta V_{H}$ and neglect their correlations. Below we summarize our results for the hadron $H$ being a pseudoscalar or vector meson, a positive-parity octet or decuplet baryon, and a negativeparity baryon, respectively.

Note that the $\rho$ and $K^{*}$ mesons as well as the negativeparity baryons are not stable for our light quark mass value and lattice volume. However, using only quark-antiquark and three-quark interpolators, we are unable to detect their decays into pairs of $p$-wave pions, pion plus kaon, and $s$-wave pion plus positive-parity baryon, respectively. As we see effective energy plateaus, we also quote results for these channels. Clearly, this needs to be digested with some caution. We also note that the disconnected quark line contribution was neglected for the $\phi$ meson.

\section{Mesons}

Several hidden charm resonances such as the $Y(4260)$ have been interpreted as tightly bound quarkonium states, embedded within light mesonic matter [21,22]. Here we follow the procedure described above to calculate the modification of the static potential, $\Delta V_{H}(r, \delta t)$, for several light mesons.

In Figs. 6, 7, 8, and 9, we show our determinations for the $\pi, K, K^{\star}$, and $\phi$ mesons, respectively, where the color coding corresponds to different values of $\delta t$ which are displaced horizontally in the plots for clarity.

In all the cases we find $\Delta V_{H}(r, \delta t)<0$. When considering the dependence on the spatial distance between the static sources, we observe a similar pattern for all the mesons; the modification of the static potential becomes more pronounced toward large distances $r$. For distances up to about $0.3 \mathrm{fm}$, we generally find $\left|\Delta V_{H}(r, \delta t)\right| \lesssim 1 \mathrm{MeV}$,

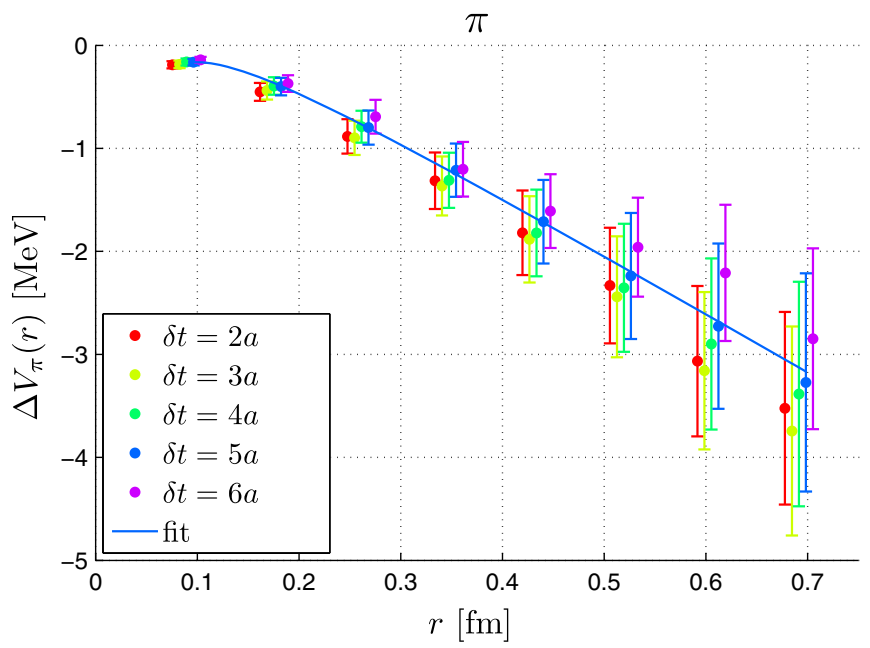

FIG. 6. The modification to the static potential in the presence of a pion, $\Delta V_{\pi}(r, \delta t)$. The color coding corresponds to different values of $\delta t$ as indicated in the legend, where the leftmost point within a group corresponds to $\delta t=2 a$. The curve shown is the result of a fit of the $\delta t=5 a$ data to Eq. (14).
PHYSICAL REVIEW D 95, 074501 (2017)

$K$

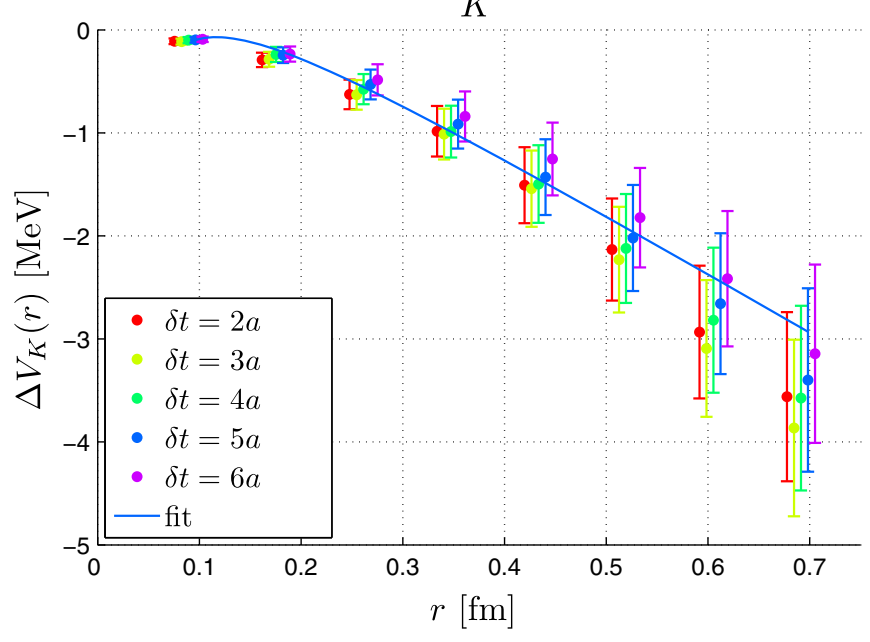

FIG. 7. The same as in Fig. 6 for the kaon.

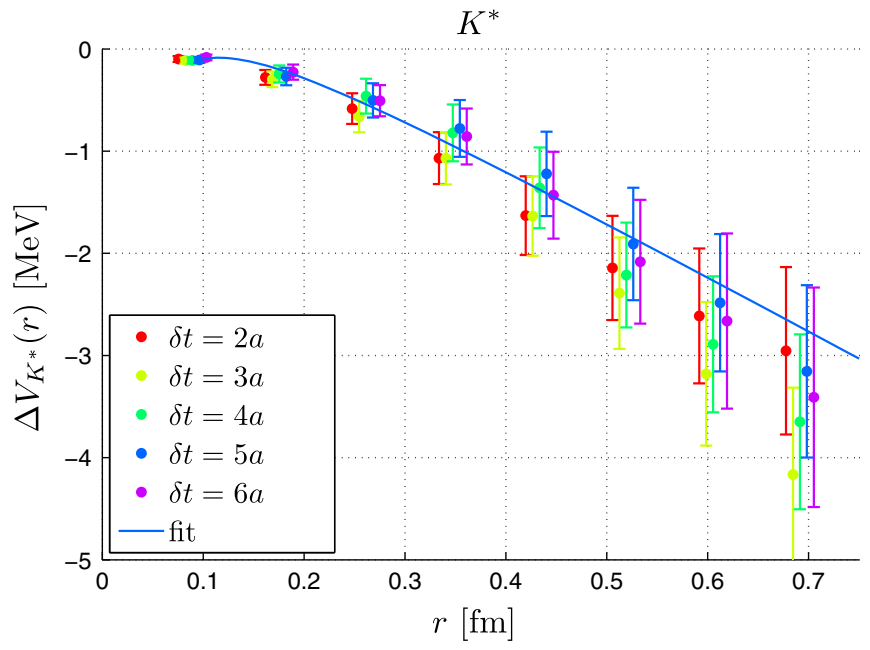

FIG. 8. The same as in Fig. 6 for the $K^{\star}$ meson.

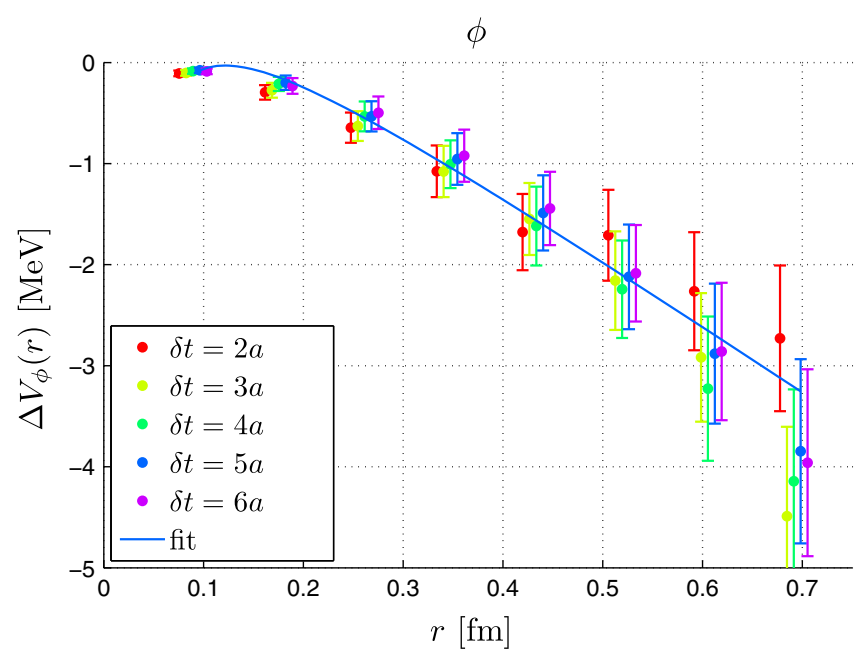

FIG. 9. The same as in Fig. 6 for the $\phi$ meson. 
TABLE I. Values of the difference in the static potential for the mesons, measured at $\delta t=5 a$. Errors are statistical and systematic, respectively.

\begin{tabular}{llllll}
\hline \hline$r / a$ & \multicolumn{1}{c}{$\Delta V_{\pi}(\mathrm{MeV})$} & \multicolumn{1}{c}{$\Delta V_{K}(\mathrm{MeV})$} & \multicolumn{1}{c}{$\Delta V_{\rho}(\mathrm{MeV})$} & \multicolumn{1}{c}{$\Delta V_{K^{\star}}(\mathrm{MeV})$} & $\Delta V_{\phi}(\mathrm{MeV})$ \\
\hline 1 & $-0.16(3)(1)$ & $-0.10(3)(1)$ & $-0.07(6)(5)$ & $-0.11(3)(3)$ & $-0.08(2)(3)$ \\
2 & $-0.40(8)(4)$ & $-0.24(8)(3)$ & $-0.17(17)(20)$ & $-0.27(8)(7)$ & $-0.22(7)(6)$ \\
3 & $-0.80(16)(19)$ & $-0.53(14)(09)$ & $-0.29(33)(56)$ & $-0.50(17)(08)$ & $-0.49(16)(9)$ \\
4 & $-1.21(26)(30)$ & $-0.91(24)(18)$ & $-0.46(52)(1.03)$ & $-0.78(28)(21)$ & $-0.85(26)(22)$ \\
5 & $-1.71(40)(56)$ & $-1.43(37)(27)$ & $-0.67(73)(1.24)$ & $-1.22(41)(45)$ & $-1.39(38)(49)$ \\
6 & $-2.24(61)(71)$ & $-2.02(51)(45)$ & $-1.33(96)(2.09)$ & $-1.91(55)(83)$ & $-2.09(52)(80)$ \\
7 & $-2.73(80)(86)$ & $-2.66(68)(71)$ & $-2.03(1.20)(3.19)$ & $-2.48(67)(1.36)$ & $-2.78(66)(1.38)$ \\
8 & $-3.27(1.06)(63)$ & $-3.40(89)(1.02)$ & $-2.77(1.46)(4.75)$ & $-3.15(84)(2.24)$ & $-3.43(84)(2.10)$ \\
\hline \hline
\end{tabular}

and at our largest shown distance of about $0.7 \mathrm{fm}$, we always find $\left|\Delta V_{H}(r, \delta t)\right| \lesssim 4 \mathrm{MeV}$. The values of $\Delta V_{H}(r)$ should be determined from the extrapolation $\delta t \rightarrow \infty$. In practice we find that all results for $\delta t \gtrsim 3 a$ agree. The numbers obtained for $\delta t=5 a$ represent a compromise between a value of $\delta t$ as large as possible and a reasonable signal-to-noise ratio. These should be considered as our final results and are displayed in Table I.

Our data are well described by the parametrization given in Eq. (14). The resulting fit parameters for the different mesons are displayed in Table II and the corresponding curves are also shown in the figures. Note that, although the fit parameters appear to indicate a somewhat different behavior for the $\rho$ meson, the data points alone, which are displayed in Table I, do not show any statistically significant deviation.

We will take the analysis one step further in Sec. VI. However, taking the above results at face value, we can already make two interesting observations. The first one is that, for identical valence quark content, there is no difference between the tendency of light pseudoscalars, such as the pion or the kaon, and vector mesons, such as the $\rho$ or the $K^{\star}$, to bind with quarkonium. The second observation is that there appears to be little or no difference increasing or decreasing the strangeness of the light mesonic matter.

\section{Positive-parity baryons}

We now turn our attention to modifications of the static potential in the presence of a positive-parity octet $\left(J^{P}=1 / 2^{+}\right)$or decuplet $\left(J^{P}=3 / 2^{+}\right)$baryon. As explained at the end of Sec. IV, in the latter case we are restricted to

TABLE II. Fit parameters for the difference of the potential for the mesons, see Eq. (14).

\begin{tabular}{lclc}
\hline \hline Meson $H$ & $\Delta \mu_{H}(\mathrm{MeV})$ & $\Delta c_{H}\left(10^{-4}\right)$ & $\Delta \sigma_{H}(\mathrm{MeV} / \mathrm{fm})$ \\
\hline$\pi$ & $0.858(39)$ & $2.30(13)$ & $-5.75(11)$ \\
$K$ & $1.167(15)$ & $3.34(52)$ & $-5.82(42)$ \\
$\rho$ & $2.28(38)$ & $6.62(1.31)$ & $-10.19(1.02)$ \\
$K^{\star}$ & $1.38(16)$ & $4.10(59)$ & $-6.47(46)$ \\
$\phi$ & $1.45(12)$ & $4.18(42)$ & $-6.67(32)$ \\
\hline \hline
\end{tabular}

employing a particular polarization to avoid mixing with $J=1 / 2$ states. In our case we project onto $J_{z}=3 / 2$ with respect to the $z$ axis. We remark that embedding charmonium states within baryons of vanishing strangeness could

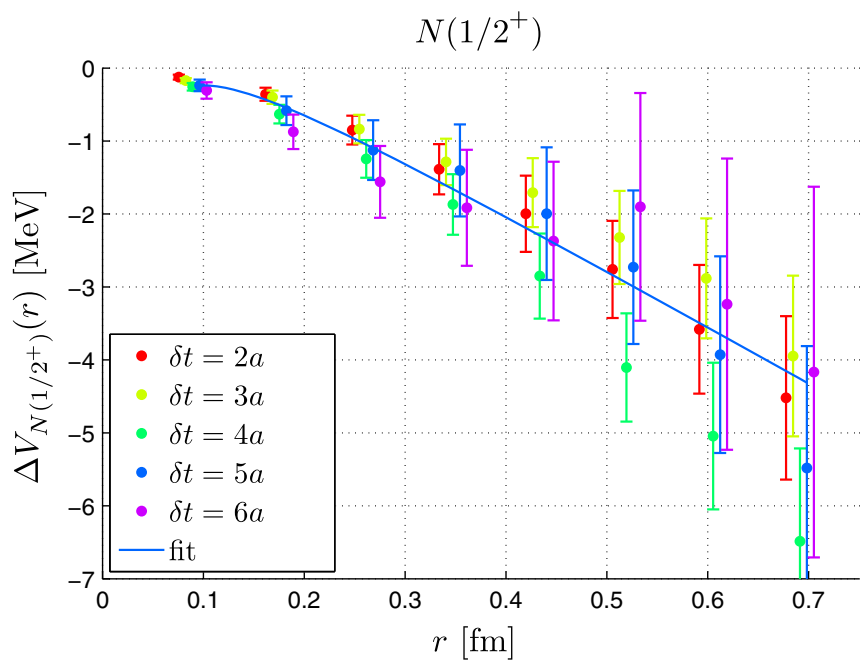

FIG. 10. The same as in Fig. 6 for the positive-parity nucleon.

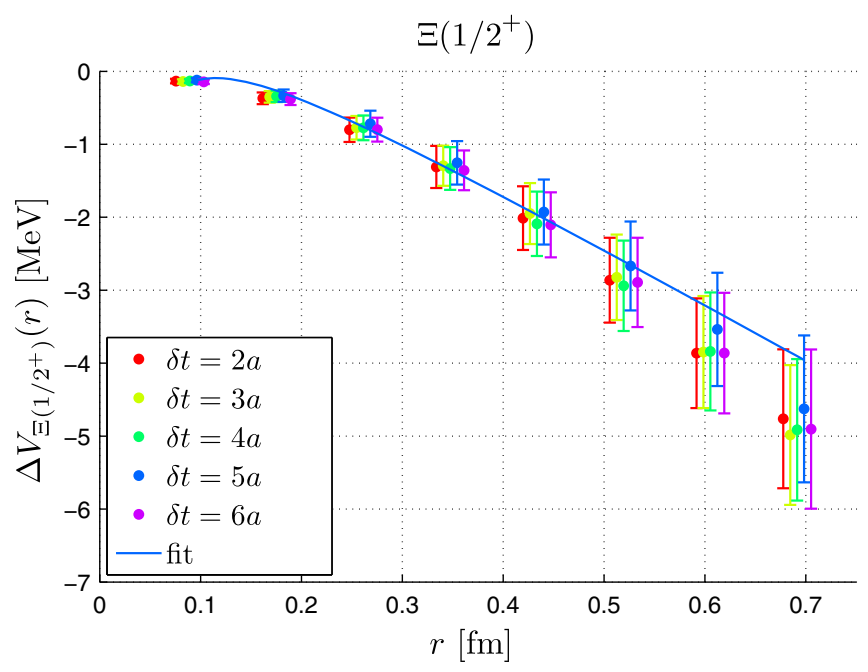

FIG. 11. The same as in Fig. 6 for the positive-parity cascade. 
HADROQUARKONIUM FROM LATTICE QCD

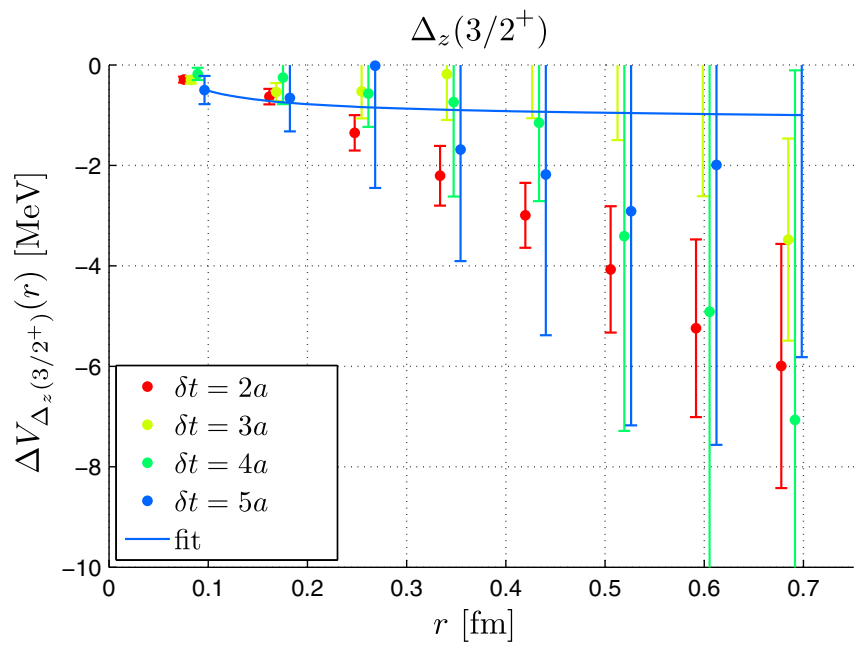

FIG. 12. The same as in Fig. 6 for the positive-parity $\Delta$ baryon. The subscript $z$ of the baryon label refers to the projection along the $\mathrm{Z}$ axis $J_{z}=3 / 2$, ensuring no mixing with $J^{P}=1 / 2^{+}$states takes place.

be an interpretation of the "pentaquark" structures that were recently reported by the LHCb Collaboration [1,2]; for examples see the last paragraph of Sec. III.

In Figs. 10, 11, 12, and 13 we show $\Delta V_{H}(r, \delta t)$ for the nucleon, the cascade $\Xi$, the $\Delta$, and the decuplet cascade $\Xi^{*}$, respectively. Again, in all the cases we observe $\Delta V_{H}(r, \delta t)<0$. The results for the positive-parity baryons are collected in Table III and are very similar to the values discussed above for the pseudoscalar and vector mesons. Note, however, that the errors of $\Delta V$ in the presence of decuplet baryons become rather large. In particular, this is
PHYSICAL REVIEW D 95, 074501 (2017)

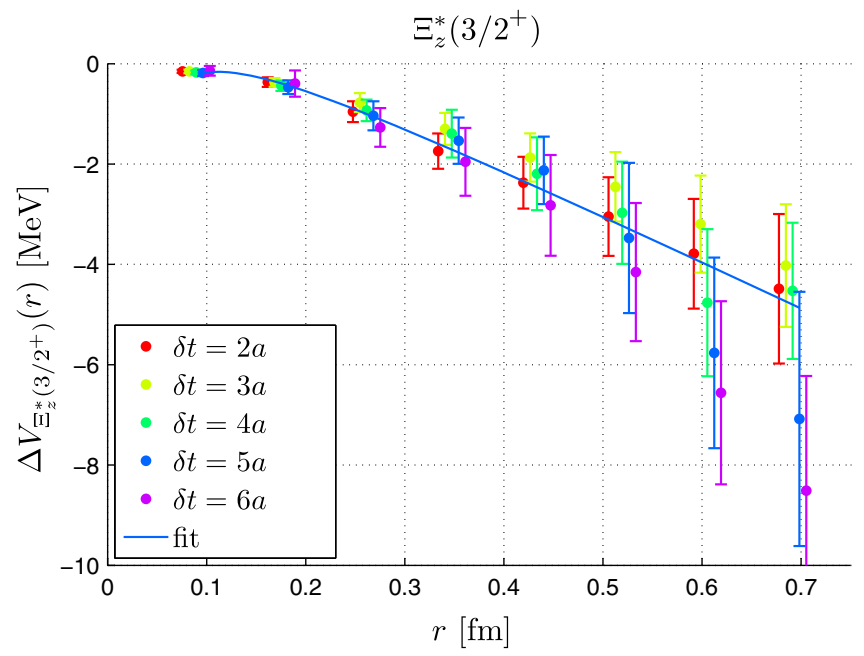

FIG. 13. The same as in Fig. 12 for the positive-parity $\Xi^{\star}$ baryon.

so for the $\Delta$, which is why in this case we only show the data up to $\delta t=5 a$. The Cornell fit parameters [Eq. (14)] are displayed in Table IV.

\section{Negative-parity baryons}

The modification of the potential in the presence of negative-parity baryons appears statistically consistent to the positive-parity case; however, due to the much larger statistical errors, we cannot exclude a more rapid decrease of $\Delta V_{H}(r)$ as a function of $r$. As examples we show in Figs. 14, 15, and 16 the results for the negative-parity

TABLE III. Values of the difference in the static potential for the positive-parity baryons, measured at $\delta t=5 a$.

\begin{tabular}{lllll}
\hline \hline$r / a$ & $\Delta V_{N\left(1 / 2^{+}\right)}(\mathrm{MeV})$ & $\Delta V_{\Sigma\left(1 / 2^{+}\right)}(\mathrm{MeV})$ & $\Delta V_{\Lambda\left(1 / 2^{+}\right)}(\mathrm{MeV})$ & $\Delta V_{\Xi\left(1 / 2^{+}\right)}(\mathrm{MeV})$ \\
\hline 1 & $-0.24(8)(13)$ & $-0.12(3)(10)$ & $-0.24(4)(5)$ & $-0.12(3)(5)$ \\
2 & $-0.58(19)(33)$ & $-0.32(9)(20)$ & $-0.60(10)(15)$ & $-0.33(8)(9)$ \\
3 & $-1.12(41)(68)$ & $-0.67(20)(38)$ & $-1.12(22)(29)$ & $-0.72(18)(15)$ \\
4 & $-1.40(63)(72)$ & $-1.22(32)(33)$ & $-1.64(35)(28)$ & $-1.25(30)(22)$ \\
5 & $-1.99(91)(67)$ & $-2.03(49)(54)$ & $-2.49(60)(46)$ & $-1.93(44)(40)$ \\
6 & $-2.73(1.05)(1.08)$ & $-2.87(68)(91)$ & $-3.21(80)(59)$ & $-2.67(61)(51)$ \\
7 & $-3.93(1.35)(1.53)$ & $-3.62(90)(1.08)$ & $-4.19(1.00)(1.30)$ & $-3.54(78)(1.00)$ \\
8 & $-5.48(1.67)(2.28)$ & $-4.40(1.16)(1.47)$ & $-5.34(1.23)(1.84)$ & $-4.63(1.01)(1.80)$ \\
\hline \hline & $\Delta V_{\Delta\left(3 / 2^{+}\right)(\mathrm{MeV})}$ & $\Delta V_{\Sigma^{*}\left(3 / 2^{+}\right)(\mathrm{MeV})}$ & $\Delta V_{\Xi^{*}\left(3 / 2^{+}\right)(\mathrm{MeV})}$ & $\Delta V_{\Omega\left(3 / 2^{+}\right)(\mathrm{MeV})}$ \\
\hline 1 & $-0.50(28)(46)$ & $-0.23(9)(7)$ & $-0.18(6)(5)$ & $-0.15(4)(5)$ \\
2 & $-0.65(66)(58)$ & $-0.49(24)(13)$ & $-0.47(14)(20)$ & $-0.40(10)(16)$ \\
3 & $-0.01(2.43)(1.29)$ & $-1.27(52)(31)$ & $-1.04(29)(48)$ & $-0.91(22)(40)$ \\
4 & $-1.68(2.22)(1.22)$ & $-1.96(84)(45)$ & $-1.53(46)(72)$ & $-1.45(37)(70)$ \\
5 & $-2.18(3.20)(3.04)$ & $-3.27(1.18)(65)$ & $-2.12(67)(99)$ & $-2.07(53)(1.22)$ \\
6 & $-2.91(4.26)(3.64)$ & $-5.33(2.81)(1.65)$ & $-3.47(1.50)(1.04)$ & $-3.31(1.00)(1.41)$ \\
7 & $-1.99(5.75)(2.12)$ & $-5.41(2.09)(1.86)$ & $-5.76(1.90)(1.87)$ & $-5.70(1.23)(2.24)$ \\
8 & $9.48(15.3)(11.4)$ & $-6.14(2.79)(2.02)$ & $-7.08(2.53)(3.02)$ & $-7.35(1.66)(3.37)$ \\
\hline \hline
\end{tabular}


TABLE IV. Fit parameters for the difference of the potential for the positive-parity baryons, see Eq. (14).

\begin{tabular}{lccl}
\hline \hline Baryon $H$ & $\Delta \mu_{H}(\mathrm{MeV})$ & $\Delta c_{H}\left(10^{-4}\right)$ & $\Delta \sigma_{H}(\mathrm{MeV} / \mathrm{fm})$ \\
\hline$N\left(1 / 2^{+}\right)$ & $1.17(37)$ & $3.21(1.30)$ & $-7.83(97)$ \\
$\Sigma\left(1 / 2^{+}\right)$ & $1.62(21)$ & $4.63(73)$ & $-7.99(60)$ \\
$\Lambda\left(1 / 2^{+}\right)$ & $1.28(20)$ & $3.46(69)$ & $-8.49(57)$ \\
$\Xi\left(1 / 2^{+}\right)$ & $1.54(19)$ & $4.32(75)$ & $-7.81(55)$ \\
$\Delta\left(3 / 2^{+}\right)$ & $-0.99(1.75)$ & $-2.22(6.16)$ & $-0.10(4.77)$ \\
$\Sigma^{\star}\left(3 / 2^{+}\right)$ & $2.15(37)$ & $6.14(1.30)$ & $-11.38(1.01)$ \\
$\Xi^{\star}\left(3 / 2^{+}\right)$ & $1.74(36)$ & $4.90(1.41)$ & $-9.40(1.03)$ \\
$\Omega\left(3 / 2^{+}\right)$ & $2.34(49)$ & $6.77(1.68)$ & $-11.02(1.41)$ \\
\hline \hline
\end{tabular}

partners of the nucleon, the cascade, and the decuplet cascade, respectively. The corresponding numerical values for $\delta t=5 a$ are displayed in Table $\mathrm{V}$ and the Cornell fit parameters in Table VI.

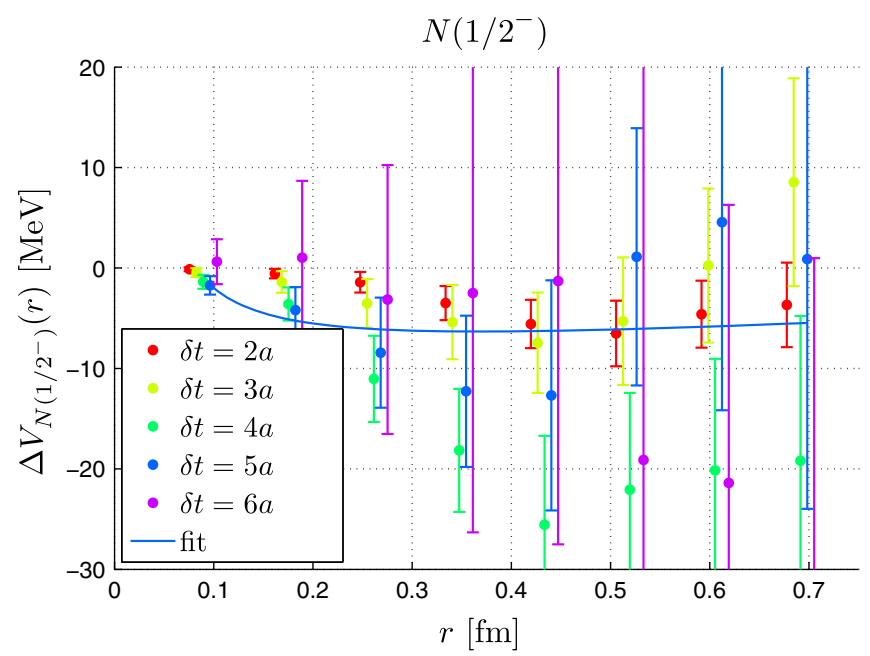

FIG. 14. The same as in Fig. 6 for the negative-parity nucleon.

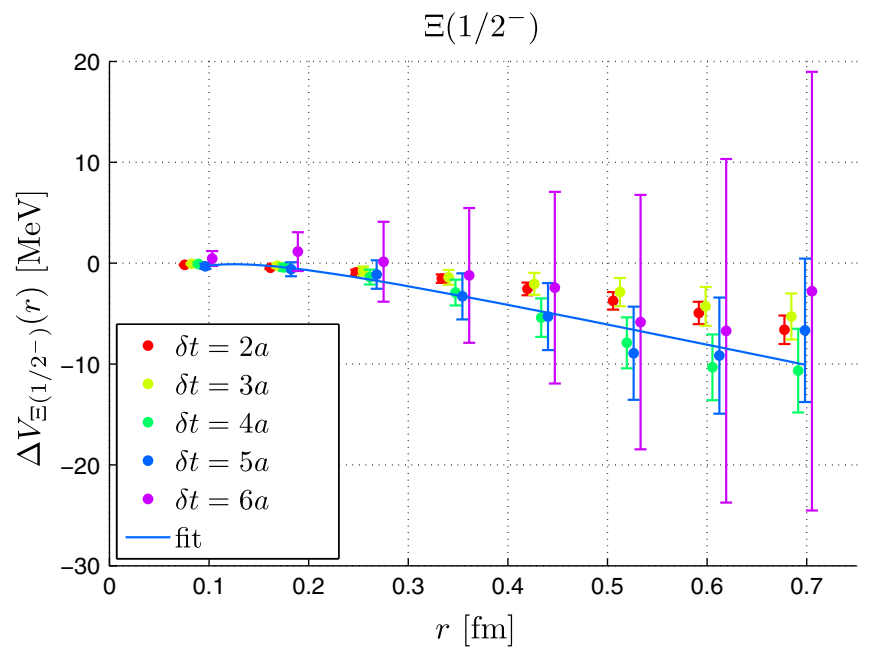

FIG. 15. The same as in Fig. 6 for the negative-parity cascade.

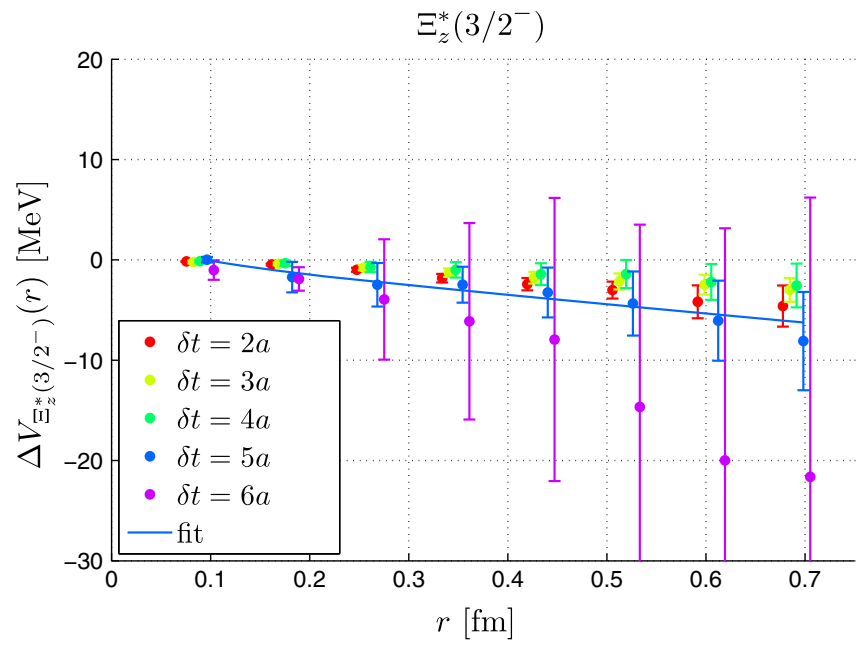

FIG. 16. The same as in Fig. 12 for the negative-parity $\Xi^{\star}$ baryon.

\section{Summary}

Regardless of meson or baryon, spin, strangeness, or parity, the modifications of the static potential are well described by the parametrization Eq. (14), with the main effects being a reduction of the linear slope and increases of the Coulomb coefficient $c$ and of the offset $\mu$. All data are consistent with a decrease of the static potential at the distance $r=0.5 \mathrm{fm}$ by about 2-3 MeV.

For $r>0.7 \mathrm{fm}$ the statistical errors grow substantially as a result of the deteriorating signal-to-noise ratio. Fortunately, larger distances exceed the size both of charmonium and of the hosting hadron and will not be relevant for the discussion of Sec. VI below. However, one may wonder if the reduction persists. In Fig. 17 we show the data for the pion, the kaon, the nucleon, and the cascade up to $r \approx 1.2 \mathrm{fm}$, a distance around which string breaking will occur $[39,61]$. The decrease of the slope appears to be robust and all large distance data points are consistent with our parametrizations. However, for the more compact pseudoscalar mesons and in particular the kaon the data suggests that above $r \approx 0.8 \mathrm{fm}$ some saturation may set in.

\section{MODIFICATION OF CHARMONIUM BINDING ENERGIES}

We have investigated how the static quark-antiquark potential changes in the presence of a light hadron. This is a well-defined observable and the results by themselves are already interesting. However, we wish to go one step further and address possible phenomenological consequences. We start with a few words of caution. When it comes to charmonia (and even for bottomonia), relativistic corrections are not small. Moreover, baryons are not particularly light in comparison to the charm quark. Therefore, for charmonia it may be doubtful if their effect 
TABLE V. Values of the difference in the static potential for the negative-parity baryons, measured at $\delta t=5 a$.

\begin{tabular}{lccrr}
\hline \hline$r / a$ & $\Delta V_{N\left(1 / 2^{-}\right)}(\mathrm{MeV})$ & $\Delta V_{\Sigma\left(1 / 2^{-}\right)}(\mathrm{MeV})$ & $\Delta V_{\Lambda\left(1 / 2^{-}\right)}(\mathrm{MeV})$ & $\Delta V_{\Xi\left(1 / 2^{-}\right)}(\mathrm{MeV})$ \\
\hline 1 & $-1.73(91)(43)$ & $-0.68(50)(77)$ & $-1.09(48)(95)$ & $-0.31(26)(29)$ \\
2 & $-4.19(2.30)(1.74)$ & $-1.46(1.27)(1.61)$ & $-2.59(1.24)(1.92)$ & $-0.60(69)(51)$ \\
3 & $-8.43(5.48)(4.19)$ & $-2.93(2.59)(3.16)$ & $-5.31(2.69)(3.68)$ & $-1.13(1.41)(58)$ \\
4 & $-12.27(7.53)(5.25)$ & $-5.12(4.06)(4.76)$ & $-8.08(4.12)(6.48)$ & $-3.29(2.28)(2.08)$ \\
5 & $-12.67(11.4)(9.74)$ & $-6.21(6.01)(6.09)$ & $-12.1(6.15)(10.1)$ & $-5.28(3.32)(3.80)$ \\
6 & $1.11(12.7)(22.9)$ & $-5.80(8.06)(5.20)$ & $-14.2(6.43)(9.63)$ & $-8.93(4.61)(6.57)$ \\
7 & $4.55(18.7)(31.8)$ & $-7.29(10.2)(5.93)$ & $-7.36(6.80)(7.91)$ & $-9.16(5.75)(6.29)$ \\
8 & $0.88(24.8)(35.4)$ & $-9.12(12.9)(7.14)$ & $-3.93(8.81)(19.5)$ & $-6.65(7.10)(5.83)$ \\
\hline \hline & & & & $\Delta V_{\Xi^{\star}\left(3 / 2^{-}\right)}(\mathrm{MeV})$ \\
\hline 1 & $\Delta V_{\Delta\left(3 / 2^{-}\right)(\mathrm{MeV})}$ & $\Delta V_{\Sigma^{\star}\left(3 / 2^{-}\right)(\mathrm{MeV})}$ & $0.01(28)(25)$ & $-0.11(20)(10)$ \\
2 & $0.98(7.20)(1.66)$ & $-0.70(1.22)(0.28)$ & $-1.72(1.52)(1.02)$ & $-0.46(47)(13)$ \\
3 & $0.07(16.7)(7.52)$ & $-3.74(4.87)(1.49)$ & $-2.47(2.17)(1.57)$ & $-0.64(96)(55)$ \\
4 & $0.97(29.2)(16.1)$ & $-8.06(5.46)(3.29)$ & $-2.47(1.79)(0.83)$ & $-0.48(1.50)(1.30)$ \\
5 & $49.3(64.4)(7.60)$ & $-13.8(8.48)(3.86)$ & $-3.26(2.48)(3.05)$ & $0.01(2.11)(2.11)$ \\
6 & $35.8(95.8)(12.5)$ & $-12.6(6.80)(12.6)$ & $-4.35(3.19)(5.40)$ & $-0.46(2.87)(2.76)$ \\
7 & $56.9(96.8)(17.2)$ & $-11.0(32.4)(4.25)$ & $-6.06(3.99)(5.70)$ & $-1.62(3.73)(2.77)$ \\
8 & $1.41(132.0)(34.6)$ & $-8.06(42.7)(9.71)$ & $-8.09(4.90)(6.33)$ & $-3.33(4.77)(3.23)$ \\
\hline \hline
\end{tabular}

can be completely integrated out in a Born-Oppenheimer or adiabatic spirit and put into the quark-antiquark interaction potential. This is less of a problem for the pion and the kaon since $M_{K} / m_{c}$ and $M_{\pi} / m_{c}$ are of similar sizes as the squared velocity $v^{2} \sim 0.3$. In what follows, we will neglect these effects.

We start from the Schrödinger equation

$\left[-\frac{\nabla^{2}}{m_{c}}+E_{H}(r)\right] \psi_{n L}^{(H)}(r, \theta, \phi)=M_{n L}^{(H)} \psi_{n L}^{(H)}(r, \theta, \phi)$,

where the reduced mass is $m_{c} / 2$ and

$$
\begin{aligned}
E_{H}(r) & =2\left(m_{c}-\delta m\right)+V_{H}(r) \\
& =2 m_{c}+v_{0}+\Delta \mu_{H}-\frac{c_{H}}{r}+\sigma_{H} r .
\end{aligned}
$$

In the second step, we have assumed the Cornell parametrization given by Eqs. (10) and (14), where we

TABLE VI. Fit parameters for the difference of the potential for the negative-parity baryons, see Eq. (14).

\begin{tabular}{lccc}
\hline \hline Baryon $H$ & $\Delta \mu_{H}(\mathrm{MeV})$ & $\Delta c_{H}\left(10^{-4}\right)$ & $\Delta \sigma_{H}(\mathrm{MeV} / \mathrm{fm})$ \\
\hline$N\left(1 / 2^{-}\right)$ & $-10.18(6.43)$ & $-3.50(22.58)$ & $5.39(17.46)$ \\
$\Sigma\left(1 / 2^{-}\right)$ & $1.88(83)$ & $4.84(2.91)$ & $-16.89(2.26)$ \\
$\Lambda\left(1 / 2^{-}\right)$ & $-0.77(3.51)$ & $-5.03(12.61)$ & $-16.92(8.93)$ \\
$\Xi\left(1 / 2^{-}\right)$ & $4.74(1.18)$ & $14.21(4.11)$ & $-20.93(3.25)$ \\
$\Delta\left(3 / 2^{-}\right)$ & $-23.1(27.6)$ & $-69.34(96.85)$ & $94.8(76.2)$ \\
$\Sigma^{\star}\left(3 / 2^{-}\right)$ & $-0.853(3.26)$ & $-12.11(11.90)$ & $-30.3(7.22)$ \\
$\Xi^{\star}\left(3 / 2^{-}\right)$ & $-0.23(1.25)$ & $-4.37(4.59)$ & $-8.92(2.61)$ \\
$\Omega\left(3 / 2^{-}\right)$ & $-0.47(62)$ & $-1.92(2.18)$ & $-0.99(1.67)$ \\
\hline \hline
\end{tabular}

set $c_{H}=c+\Delta c_{H}$ and $\sigma_{H}=\sigma+\Delta \sigma_{H}$. The parameters $\Delta \mu_{H}, \Delta c_{H}$, and $\Delta \sigma_{H}$ specify the modifications of the constant, the Coulomb, and the linear terms, respectively, obtained from the Cornell fits to $\Delta V_{H}(r)=V_{H}(r)-V_{0}(r)$ carried out in the previous section.

The Cornell parametrization is not valid at large distances due to string breaking effects $[39,61]$ or at small distances where one would expect the coefficient $c_{H}$ to run with the scale $r$. However, we are only interested in mass differences $\Delta M_{n L}^{(H)}=M_{n L}^{(H)}-M_{n L}^{(0)}$ between a charmonium state with radial and angular momentum quantum numbers $n$ and $L$ respectively, in the presence of a hadron $H$, relative

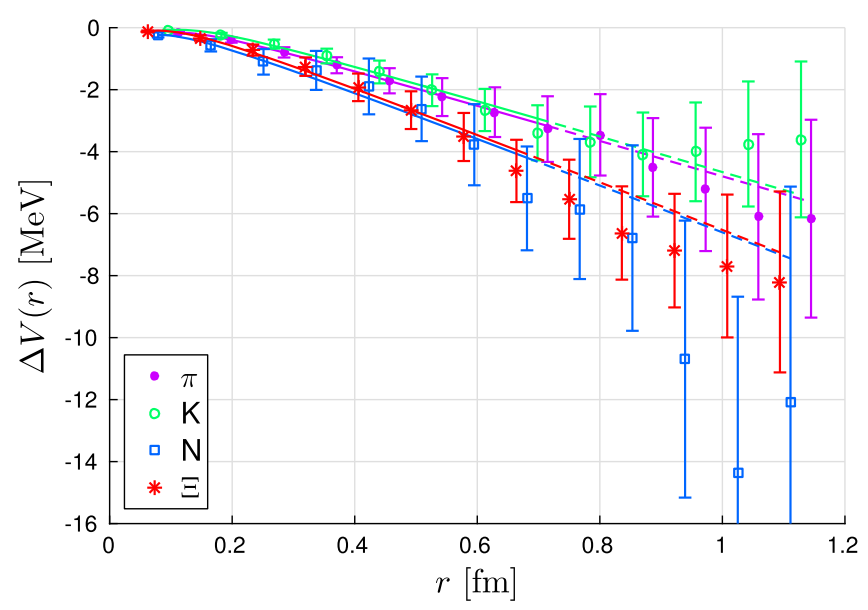

FIG. 17. The difference in the static potential for the pion, the kaon, and the positive-parity nucleon and cascade, measured at $\delta t=5 a$, up to a distance of $1.2 \mathrm{fm}$. The curves correspond to the parametrization Eq. (14) with the parameters (obtained by fitting the $r<0.7 \mathrm{fm}$ data points) displayed in Tables II and IV. 
to the same state in the vacuum. We expect such corrections to affect both masses in similar ways, and therefore to cancel from these differences. The coefficients $\Delta \mu_{H}, c_{H}$, and $\sigma_{H}$ are taken from the fits performed in the previous section, while the mass parameter $m_{c}$ and the offset $v_{0}=$ $\mu-2 \delta m$ have to be fixed by matching the energy levels $M_{n L}=M_{n L}^{(0)}$, obtained from solving the above Schrödinger equation, to experiment.

Due to the approximations made, our discussion can only be qualitative and hence we neglect our statistical and systematic uncertainties. The central values for the parameters from the Cornell fit to the static potential in the vacuum read [see also Eq. (11)]

$$
\sigma=0.0335 a^{-2} \approx(423 \mathrm{MeV})^{2}, \quad c=0.468 .
$$

Numerically solving the Schrödinger equation and adjusting $m_{c}$ and $v_{0}$ so that we reproduce the spin-averaged $1 S$ and $2 S$ charmonium levels, we find

$$
m_{c}=1269 \mathrm{MeV}, \quad v_{0}=113 \mathrm{MeV} .
$$

From Table VII, we see that the above parameters indeed reproduce the experimental $1 S$ and $2 S$ levels; however, we underestimate the $1 P$ mass by $42 \mathrm{MeV}$. This is due to a combination of overestimating the value of the wave function at the origin, as we neglected running coupling effects, and relativistic corrections [63]; within our approximations, it is not possible to simultaneously reproduce all spin-independent mass splittings within an accuracy better than about $10 \%$.

A negative value of $\Delta M_{n L}^{(H)}$ means that embedding a charmonium state within the hadron $H$ is energetically favorable, which we interpret as attraction. Unlike in the hydrogen case, the potential is only bound from above by the $D \bar{D}$ threshold and so it may not be entirely obvious

TABLE VII. Masses and mass differences of spin-averaged states in $\mathrm{MeV}$ taken from experiment [43] and from solving the Schrödinger equation using the Cornell parametrization of our lattice results.

\begin{tabular}{lrrc}
\hline \hline Mass/Mass difference & $1 S(\mathrm{MeV})$ & $1 P(\mathrm{MeV})$ & $2 S(\mathrm{MeV})$ \\
\hline$M_{n L}$ (experiment) & 3068.6 & 3525.3 & 3674.4 \\
$M_{n L}$ (Schrödinger) & 3068.6 & 3483.3 & 3674.4 \\
$\Delta M^{(\pi)}$ & -1.7 & -3.1 & -4.0 \\
$\Delta M^{(K)}$ & -1.5 & -2.9 & -3.8 \\
$\Delta M^{(\rho)}$ & -2.5 & -4.9 & -6.5 \\
$\Delta M^{\left(K^{*}\right)}$ & -1.6 & -3.2 & -4.2 \\
$\Delta M^{(\phi)}$ & -1.6 & -3.2 & -4.3 \\
$\Delta M^{(N)}$ & -2.4 & -4.3 & -5.5 \\
$\Delta M^{(\Xi)}$ & -2.0 & -3.9 & -5.1 \\
$\Delta M^{(\Delta)}$ & -0.9 & -1.0 & -1.0 \\
$\Delta M^{\left(\Xi^{*}\right)}$ & -2.6 & -4.8 & -6.3 \\
\hline \hline
\end{tabular}

whether a negative $\Delta V_{H}(r)$ results in a positive or a negative shift of the charmonium mass. On one hand, a lower $V_{H}$ results in a lower $E_{H}$ and therefore in a smaller $M_{n L}^{(H)}$ mass. On the other hand, the slope is reduced, resulting in a more extended and less strongly bound wave function.

Before numerically solving the Schrödinger equation we investigate a toy model with a purely linear potential $V(r)=\sigma r$. The virial theorem then gives a kinetic energy

$$
2\langle T\rangle=\langle r \mathrm{~d} V / \mathrm{d} r\rangle=\sigma\langle r\rangle=2 M-2 \sigma\langle r\rangle,
$$

where we used $M=\langle T\rangle+\langle V\rangle=\langle T\rangle+\sigma\langle r\rangle$. This means that $\langle r\rangle=2 M /(3 \sigma)$. The Feynman-Hellmann theorem then gives

$$
\frac{\partial M}{\partial \sigma}=\left\langle\frac{\partial H}{\partial \sigma}\right\rangle=\langle r\rangle=\frac{2 M}{3 \sigma},
$$

i.e.

$$
\Delta M^{(H)}=\left.\left(\sigma_{H}-\sigma\right) \frac{\partial M}{\partial \sigma}\right|_{\sigma=\sigma_{0}}=\frac{2 \sigma_{H}}{3 \sigma_{0}} M^{(0)},
$$

where $M^{(H)}=M\left(\sigma_{H}\right)$. Therefore, we expect the part of the mass which is due to the interaction, $M-2\left(m_{c}-\delta m\right)$, to be lowered by a factor $2 \sigma_{H} /(3 \sigma)$, which for our data typically amounts to about $0.4 \%$. As we have neglected Coulomb interactions, we should also neglect the self-energy $\delta m$. Then, using the $m_{c}$ value of Eq. (19) and $M_{1 S}=3069 \mathrm{MeV}$, this difference gives $530 \mathrm{MeV}$. So, for the $1 S$ state, we expect an attraction $\Delta M_{1 S}^{(H)} \approx-2 \mathrm{MeV}$. Using the experimental $1 P-1 S$ and $2 S-1 S$ differences lowers this to $\Delta M_{1 P}^{(H)} \approx$ $-3.9 \mathrm{MeV}$ and $\Delta M_{2 S}^{(H)} \approx-4.5 \mathrm{MeV}$, respectively.

We now solve the Schrödinger equation numerically for the mesons and for some of the positive-parity baryons, using the parameter values of Eqs. (18) and (19), together with $\Delta \mu_{H}, \Delta c_{H}$, and $\Delta \sigma_{H}$ obtained from the fits to $\Delta V_{H}(r)$, see Tables II and IV. The results are collected in Table VII. Indeed, the masses in all the channels shown are lowered by amounts that are in qualitative agreement with the considerations from the virial and Feynman-Hellmann theorems above, and the effect becomes larger for spatially more extended charmonia. Note that the potentials for the $\rho$ meson and the $\Delta$ baryon have relatively large errors. Therefore, the resulting mass shifts statistically agree with those shown for the $K^{*}$ and the $\Xi^{*}$, respectively.

In Ref. [35], a charmonium-nucleon bound state energy of $-20 \mathrm{MeV}$ was reported-a factor of 8 larger than our result. The light quark mass employed in that study was approximately 13 times larger than the one we use here. However, as one can see from Table VII, if we replace the nucleon by the cascade that contains two strange quarks, which are 8 times heavier than our light quark, the binding 
appears to become even weaker, albeit by a statistically insignificant difference.

We found that, within the approximations made, the binding of the charmonium $1 S$ state becomes stronger by values ranging from $-1 \mathrm{MeV}$ to $-2.5 \mathrm{MeV}$. For the $2 S$ state this effect increases to $-1 \mathrm{MeV}$ to $-6.5 \mathrm{MeV}$. Such estimates will be more reliable for bottomonia where relativistic and $m_{H} / m_{b}$ corrections are smaller. However, these states are also less extended spatially and $V_{0}(r)$ is most strongly modified towards large distances. This means that the mass shifts induced by the presence of a light hadron will be even smaller in the bottomonium case since charmonium and bottomonium binding energies $\sim m_{Q} v^{2}$ are of similar sizes.

\section{SUMMARY AND OUTLOOK}

Studying charmonium resonances above strong decay thresholds poses a considerable challenge to lattice QCD. In most cases not only radial excitations of the charm quark-antiquark system need to be resolved but also several decay channels open up, at least near the physical values of the light quark mass. Some of the relevant thresholds involve the scattering of three and more hadrons. In this case even the required methodology is under active development-for recent progress in this direction, see Refs. [64-69]. In view of this, testing specific models or making assumptions in certain limiting cases represents a viable alternative and may provide at least some firstprinciples insight into the nature of exotic bound states containing hidden charm.

Here we have investigated in the heavy quark limit the hadro-quarkonium picture [22], which assumes quarkonium can be bound inside the core of a light hadron. We employed a single CLS [44] ensemble with $N_{f}=2+1$ flavors of nonperturbatively order- $a$-improved Wilson quarks at a lattice spacing $a \approx 0.085 \mathrm{fm}$. The pion and kaon masses are approximately $223 \mathrm{MeV}$ and $476 \mathrm{MeV}$, respectively; i.e., the light quark mass is by a factor of about 2.7 larger than in nature. Our approach for testing this picture was first to determine the potential between a pair of static sources, approximating a heavy quark-antiquark pair, in the absence of the hadron. Assuming the nonrelativistic limit, the Schrödinger equation can then be solved with this potential in order to obtain (spin-averaged) quarkonium energy levels. This approach can be extended systematically, adding $v^{2}$ corrections, to include heavy quark spin and momentum dependent effects [70-75]. Making the additional assumption that the heavy quark mass is much larger than the mass of the light hadron, the effect of the light hadron onto the quarkonium can also be integrated out adiabatically and cast into the quark-antiquark interaction potential.

We calculated such potentials in the background of a hadron $H$ for a variety of pseudoscalar and vector mesons, octet and decuplet baryons, and their negative-parity partners. Of particular interest are the differences $\Delta V_{H}(r)$, relative to the potential in the vacuum. Solving the Schrödinger equation with the modified potential and comparing the outcome to the results obtained in vacuo provides an indication of the strength of the binding between the host hadron and the quarkonium, at least in the heavy quark limit. In principle this approach can also be extended, including mass-dependent corrections and interactions between the spins of the hadron and the heavy quarks. As the effects we detected were quite small, we have, however, no immediate plans of pursuing this line of research.

Resolving very small energy differences was possible by employing a large number of sources on 1552 gauge configurations, corresponding to over 6000 molecular dynamics units of the hybrid Monte Carlo algorithm. For all the light mesons, namely the $\pi, K, \rho, K^{\star}$, and $\phi$, as well as the baryons we considered, namely the $N, \Sigma, \Lambda$, $\Xi, \Delta, \Sigma^{*}, \Xi^{*}$, and $\Omega$ of both parities, we found $\Delta V_{H}(r)<0$, suggesting a tendency to bind. The main effect could be quantified as a reduction of the linear slope of the potential. At a distance of $0.5 \mathrm{fm}$ the potential was lowered by only 2-3 MeV for all these hadrons. Increasing the strangeness resulted in smaller statistical errors but differences between the investigated hadrons were not significant. Translating the modification of the potential into energy levels by solving the Schrödinger equation suggested values for the finite volume binding energy of $1 S$ charmonium ranging from -1 to $-2.5 \mathrm{MeV}$ and $2 S$ charmonium from -1 to $-6.5 \mathrm{MeV}$, see Table VII. These effects should be even smaller for bottomonia that are most sensitive to modifications of the potential at very short distances.

These binding energies are similar in size to that of deuterium and may be hard to reconcile with the hadroquarkonium picture where the quarkonium is thought to be localized inside the light hadron which has a size $\lesssim 1 \mathrm{fm}$. Therefore, in the heavy quark limit, which should at least apply to bottomonia, this may not be a viable picture. We cannot exclude, however, different mechanisms to stabilize hadrocharmonia such as relativistic corrections or corrections due to the mass of the hosting hadron.

The spatial lattice extent $L \approx 4.6 / M_{\pi} \approx 4.1 \mathrm{fm}$ was not only large relative to the inverse pion mass but also in comparison to the size of a light hadron or a quarkonium state; however, the observed effects were very small. Hence, a finite-volume study (see, e.g., Ref. [33]) is required to establish if the reported binding energies survive the infinite-volume limit. Simulations on different volumes, and also injecting momentum to enable a scattering study, are ongoing; see Ref. [76] for preliminary results. Until these more extensive investigations are concluded, we cannot exclude the possibility that no bound state or resonance exists. Therefore, the binding energies presented here should only be considered as upper limits. 


\section{ACKNOWLEDGMENTS}

This work was supported by the Deutsche Forschungsgemeinschaft (DFG) Grant No. SFB/TRR 55. G. M. acknowledges support from the Herchel Smith Fund at the University of Cambridge and the DFG under Contract No. KN 947/1-2. The ensemble C101 was generated by CLS [44] employing the openQCD $[45,46]$ software and using computer time provided by PRACE (Partnership for Advanced Computing in Europe, http:// www.prace-ri.eu) on Fermi at CINECA Bologna and on SuperMUC at Leibniz Supercomputing Centre Munich. An additional stream was generated on the "Clover" Cluster of the Mainz Helmholtz Institute. Analysis was performed on the SFB/TRR 55 QPACE 2 [77] Xeon-Phi installation at
Regensburg and on the Stromboli cluster in Wuppertal. We used the CHROMA [78] software package along with the LibHadronAnalysis library and the multigrid solver implementation of Ref. [79] (see also Refs. [80-82]) to generate hadronic two-point functions. Wilson loops were generated using Björn Leder's WLOOP package [50,51]. For the error analysis we used the software of the ALPHA collaboration $[57,58]$ available at http://www-zeuthen.desy.de/alpha/. We thank Peter Georg, Benjamin Gläßle, and Daniel Richtmann for support. Last but not least we thank all our CLS colleagues and in particular Dalibor Djukanovic, Georg Engel, and Leonardo Giusti for generating the C101 ensemble and Stefan Schaefer for coordinating the CLS simulations.
[1] R. Aaij et al. (LHCb Collaboration), Observation of $J / \psi \rho$ Resonances Consistent with Pentaquark States in $\Lambda_{b}^{0} \rightarrow$ $J / \psi K^{-} p$ Decays, Phys. Rev. Lett. 115, 072001 (2015).

[2] R. Aaij et al. (LHCb Collaboration), Model-Independent Evidence for $J / \psi p$ Contributions to $\Lambda_{b}^{0} \rightarrow J / \psi p K^{-}$ Decays, Phys. Rev. Lett. 117, 082002 (2016).

[3] A. P. Szczepaniak, Dalitz plot distributions in presence of triangle singularities, Phys. Lett. B 757, 61 (2016).

[4] F.-K. Guo, U.-G. Meißner, J. Nieves, and Z. Yang, Remarks on the $P_{c}$ structures and triangle singularities, Eur. Phys. J. A 52, 318 (2016).

[5] N. Brambilla et al. (Quarkonium Working Group Collaboration), Heavy quarkonium: Progress, puzzles, and opportunities, Eur. Phys. J. C 71, 1534 (2011).

[6] H.-X. Chen, W. Chen, X. Liu, and S.-L. Zhu, The hiddencharm pentaquark and tetraquark states, Phys. Rep. 639, 1 (2016).

[7] R. L. Jaffe, Multi-quark hadrons. 1. The phenomenology of $q q \bar{q} \bar{q}$ mesons, Phys. Rev. D 15, 267 (1977).

[8] J. D. Weinstein and N. Isgur, Do Multi-Quark Hadrons Exist?, Phys. Rev. Lett. 48, 659 (1982).

[9] L. Maiani, A. D. Polosa, and V. Riquer, Indications of a Four-Quark Structure for the $X(3872)$ and $X(3876)$ Particles from Recent Belle and BABAR Data, Phys. Rev. Lett. 99, 182003 (2007).

[10] S. J. Brodsky, D. S. Hwang, and R. F. Lebed, Dynamical Picture for the Formation and Decay of the Exotic XYZ Mesons, Phys. Rev. Lett. 113, 112001 (2014).

[11] R. F. Lebed, How often do diquarks form? A very simple model, Phys. Rev. D 94, 034039 (2016).

[12] M. B. Voloshin and L. B. Okun, Hydronic molecules and the charmonium atom, Pis'ma Zh. Eksp. Teor. Fiz. 23, 369 (1976) [JETP Lett. 23, 333 (1976)].

[13] A. De Rújula, H. Georgi, and S. L. Glashow, Molecular Charmonium: A New Spectroscopy?, Phys. Rev. Lett. 38, 317 (1977).

[14] V. A. Novikov, L. B. Okun, M. A. Shifman, A. I. Vainshtein, M. B. Voloshin, and V.I. Zakharov, Charmonium and gluons: Basic experimental facts and theoretical introduction, Phys. Rep. 41, 1 (1978).

[15] N. A. Törnqvist, From the deuteron to deusons, an analysis of deuteron-like meson meson bound states, Z. Phys. C 61, 525 (1994).

[16] F. Close, C. Downum, and C. E. Thomas, Novel charmonium and bottomonium spectroscopies due to deeply bound hadronic molecules from single pion exchange, Phys. Rev. D 81, 074033 (2010).

[17] T. Barnes, Colored quark and gluon constituents in the MIT bag model, Nucl. Phys. B158, 171 (1979).

[18] T. Barnes and F. E. Close, A light exotic $q \bar{q} g$ hermaphrodite meson?, Phys. Lett. 116B, 365 (1982).

[19] M. S. Chanowitz and S. R. Sharpe, Hybrids: Mixed states of quarks and gluons, Nucl. Phys. B222, 211 (1983); Erratum, Nucl. Phys. B228, 588(E) (1983).

[20] N. Isgur, R. Kokoski, and J. Paton, Gluonic Excitations of Mesons: Why They Are Missing and Where to Find Them, Phys. Rev. Lett. 54, 869 (1985); AIP Conf. Proc. 132, 242 (1985).

[21] X. Li and M. B. Voloshin, $Y(4260)$ and $Y(4360)$ as mixed hadrocharmonium, Mod. Phys. Lett. A 29, 1450060 (2014).

[22] S. Dubynskiy and M. B. Voloshin, Hadro-charmonium, Phys. Lett. B 666, 344 (2008).

[23] M. Lüscher, Two particle states on a torus, and their relation to the scattering matrix, Nucl. Phys. B354, 531 (1991).

[24] S. Prelovsek, in 7th International Workshop on Charm Physics (Charm 2015), Detroit, MI, USA, 2015 (2015), https://arxiv.org/abs/1508.07322.

[25] W. E. Caswell and G. P. Lepage, Effective Lagrangians for bound state problems in QED, QCD, and other field theories, Phys. Lett. 167B, 437 (1986).

[26] A. Pineda and J. Soto, Effective field theory for ultrasoft momenta in NRQCD and NRQED, Nucl. Phys. B, Proc. Suppl. 64, 428 (1998).

[27] N. Brambilla, A. Pineda, J. Soto, and A. Vairo, Potential NRQCD: An effective theory for heavy quarkonium, Nucl. Phys. B566, 275 (2000). 
[28] N. Brambilla, G. Krein, J. T. Castellà, and A. Vairo, Longrange properties of $1 S$ bottomonium states, Phys. Rev. D 93, 054002 (2016).

[29] S. J. Brodsky, I. A. Schmidt, and G. F. de Téramond, Nuclear Bound Quarkonium, Phys. Rev. Lett. 64, 1011 (1990).

[30] A. B. Kaidalov and P. E. Volkovitsky, Heavy Quarkonia Interactions with Nucleons and Nuclei, Phys. Rev. Lett. 69, 3155 (1992).

[31] D. A. Wasson, Comment on, "Nuclear Bound Quarkonium", Phys. Rev. Lett. 67, 2237 (1991).

[32] M. E. Luke, A. V. Manohar, and M. J. Savage, A QCD calculation of the interaction of quarkonium with nuclei, Phys. Lett. B 288, 355 (1992).

[33] K. Yokokawa, S. Sasaki, T. Hatsuda, and A. Hayashigaki, First lattice study of low-energy charmonium-hadron interaction, Phys. Rev. D 74, 034504 (2006).

[34] L. Liu, H.-W. Lin, and K. Orginos, Charmed hadron interactions, Proc. Sci. LATTICE2008 (2008) 112.

[35] S. R. Beane, E. Chang, S. D. Cohen, W. Detmold, H.-W. Lin, K. Orginos, A. Parreño, and M. J. Savage (NPLQCD Collaboration), Quarkonium-nucleus bound states from Lattice QCD, Phys. Rev. D 91, 114503 (2015).

[36] T. Kawanai and S. Sasaki, Charmonium-nucleon potential from lattice QCD, Phys. Rev. D 82, 091501 (2010).

[37] D. G. Richards, D. K. Sinclair, and D. W. Sivers, Lattice QCD simulation of meson exchange forces, Phys. Rev. D 42, 3191 (1990).

[38] P. Pennanen, C. Michael, and A. M. Green (UKQCD Collaboration), Interactions of heavy light mesons, Nucl. Phys. B, Proc. Suppl. 83-84, 200 (2000).

[39] G. S. Bali, H. Neff, T. Düssel, T. Lippert, and K. Schilling (SESAM Collaboration), Observation of string breaking in QCD, Phys. Rev. D 71, 114513 (2005).

[40] G. Bali and M. Hetzenegger (QCDSF Collaboration), Static-light meson-meson potentials, Proc. Sci. LATTICE2010 (2010) 142.

[41] A. Peters, P. Bicudo, K. Cichy, and M. Wagner, Investigation of $B \bar{B}$ four-quark systems using lattice QCD, J. Phys. Conf. Ser. 742, 012006 (2016).

[42] G. Bali and M. Hetzenegger (QCDSF Collaboration), Potentials between pairs of static-light mesons, Proc. Sci. LATTICE2011 (2011) 123.

[43] K. A. Olive et al. (Particle Data Group Collaboration), Review of Particle Physics, Chin. Phys. C 38, 090001 (2014).

[44] M. Bruno et al. (CLS Collaboration), Simulation of QCD with $N_{f}=2+1$ flavors of non-perturbatively improved Wilson fermions, J. High Energy Phys. 02 (2015) 043.

[45] M. Lüscher et al., http://luscher.web.cern.ch/luscher/ openQCD/, 2016.

[46] M. Lüscher and S. Schaefer, Lattice QCD with open boundary conditions and twisted-mass reweighting, Comput. Phys. Commun. 184, 519 (2013).

[47] M. Lüscher, Properties and uses of the Wilson flow in Lattice QCD, J. High Energy Phys. 08 (2010) 071; Erratum, J. High Energy Phys. 03 (2014) 092(E).

[48] G. S. Bali, E. E. Scholz, J. Simeth, and W. Söldner (RQCD Collaboration), Lattice simulations with $N_{f}=2+1$ improved Wilson fermions at a fixed strange quark mass, Phys. Rev. D 94, 074501 (2016).
[49] S. Borsányi et al. (BMW Collaboration), High-precision scale setting in lattice QCD, J. High Energy Phys. 09 (2012) 010.

[50] B. Leder, https://github.com/bjoern-leder/wloop/, 2016.

[51] M. Donnellan, F. Knechtli, B. Leder, and R. Sommer (ALPHA Collaboration), Determination of the static potential with dynamical fermions, Nucl. Phys. B849, 45 (2011).

[52] A. Hasenfratz and F. Knechtli, Flavor symmetry and the static potential with hypercubic blocking, Phys. Rev. D 64, 034504 (2001).

[53] M. D. Morte, S. Dürr, J. Heitger, H. Molke, J. Rolf, A. Shindler, and R. Sommer (ALPHA Collaboration), Lattice HQET with exponentially improved statistical precision, Phys. Lett. B 581, 93 (2004); Erratum, Phys. Lett. B 612, 313(E) (2005).

[54] A. Hasenfratz, R. Hoffmann, and F. Knechtli, The static potential with hypercubic blocking, Nucl. Phys. B, Proc. Suppl. 106-107, 418 (2002).

[55] M. D. Morte, A. Shindler, and R. Sommer (ALPHA Collaboration), On lattice actions for static quarks, J. High Energy Phys. 08 (2005) 051.

[56] A. Grimbach, D. Guazzini, F. Knechtli, and F. Palombi (ALPHA Collaboration), $O(a)$ improvement of the HYP static axial and vector currents at one-loop order of perturbation theory, J. High Energy Phys. 03 (2008) 039.

[57] U. Wolff (ALPHA Collaboration), Monte Carlo errors with less errors, Comput. Phys. Commun. 156, 143 (2004).

[58] S. Schaefer, R. Sommer, and F. Virotta (ALPHA Collaboration), Critical slowing down and error analysis in lattice QCD simulations, Nucl. Phys. B845, 93 (2011).

[59] S. Necco and R. Sommer, The $N_{f}=0$ heavy quark potential from short to intermediate distances, Nucl. Phys. B622, 328 (2002).

[60] E. Eichten, K. Gottfried, T. Kinoshita, J. B. Kogut, K. D. Lane, and T.-M. Yan, The Spectrum of Charmonium, Phys. Rev. Lett. 34, 369 (1975); Erratum, Phys. Rev. Lett. 36, 1276(E) (1976).

[61] V. Koch, J. Bulava, B. Hörz, F. Knechtli, G. Moir, C. Morningstar, and M. Peardon, Towards string breaking with $2+1$ dynamical fermions using the stochastic $\mathrm{LapH}$ method, Proc. Sci. LATTICE2015 (2016) 100.

[62] R. Sommer, A new way to set the energy scale in lattice gauge theories and its applications to the static force and $\alpha_{s}$ in SU(2) Yang-Mills theory, Nucl. Phys. B411, 839 (1994).

[63] G. S. Bali and P. Boyle, A lattice potential investigation of quark mass and volume dependence of the $\Upsilon$ spectrum, Phys. Rev. D 59, 114504 (1999).

[64] S. Kreuzer and H.-W. Hammer, On the modification of the Efimov spectrum in a finite cubic box, Eur. Phys. J. A 43, 229 (2010).

[65] K. Polejaeva and A. Rusetsky, Three particles in a finite volume, Eur. Phys. J. A 48, 67 (2012).

[66] R. A. Briceño and Z. Davoudi, Three-particle scattering amplitudes from a finite volume formalism, Phys. Rev. D 87, 094507 (2013).

[67] U.-G. Meißner, G. Ríos, and A. Rusetsky, Spectrum of Three-Body Bound States in a Finite Volume, Phys. Rev. Lett. 114, 091602 (2015); Erratum, Phys. Rev. Lett. 117, 069902(E) (2016). 
[68] M. T. Hansen and S. R. Sharpe, Expressing the threeparticle finite-volume spectrum in terms of the three-tothree scattering amplitude, Phys. Rev. D 92, 114509 (2015).

[69] M. T. Hansen and S. R. Sharpe, Threshold expansion of the three-particle quantization condition, Phys. Rev. D 93, 096006 (2016).

[70] E. Eichten and F. L. Feinberg, Spin dependent forces in QCD, Phys. Rev. D 23, 2724 (1981).

[71] A. Barchielli, N. Brambilla, and G. M. Prosperi, Relativistic corrections to the quark-antiquark potential and the quarkonium spectrum, Nuovo Cimento Soc. Ital. Fis. 103A, 59 (1990).

[72] G. S. Bali, K. Schilling, and A. Wachter, Complete $O\left(v^{2}\right)$ corrections to the static interquark potential from $\mathrm{SU}(3)$ gauge theory, Phys. Rev. D 56, 2566 (1997).

[73] G. S. Bali, QCD forces and heavy quark bound states, Phys. Rep. 343, 1 (2001).

[74] N. Brambilla, A. Pineda, J. Soto, and A. Vairo, Effective field theories for heavy quarkonium, Rev. Mod. Phys. 77, 1423 (2005).

[75] Y. Koma and M. Koma, Spin-dependent potentials from lattice QCD, Nucl. Phys. B769, 79 (2007).
[76] F. Knechtli, M. Alberti, G. S. Bali, S. Collins, G. Moir, and W. Söldner, Testing the hadro-quarkonium model on the lattice, Proc. Sci. LATTICE2016 (2007) 113.

[77] P. Arts et al., QPACE 2 and domain decomposition on the Intel Xeon Phi, Proc. Sci. LATTICE2014 (2015) 021.

[78] R. G. Edwards and B. Joó (SciDAC, LHP Collaboration and UKQCD Collaborations), , The Chroma software system for lattice QCD, Nucl. Phys. B, Proc. Suppl. 140, 832 (2005).

[79] S. Heybrock, M. Rottmann, P. Georg, and T. Wettig, Adaptive algebraic multigrid on SIMD architectures, Proc. Sci. LATTICE2015 (2016) 036.

[80] D. Richtmann, Simon Heybrock, and Tilo Wettig, Multiple right-hand-side setup for the DD- $\alpha \mathrm{AMG}$, Proc. Sci. LATTICE2015 (2016) 035.

[81] S. Heybrock, B. Joó, D. D. Kalamkar, M. Smelyanskiy, K. Vaidyanathan, T. Wettig, and P. Dubey, in SC '14: Proceedings of the International Conference for High Performance Computing, Networking, Storage and Analysis, New Orleans, LA, USA, 2014 (IEEE Press, Piscataway, NJ, 2014), p. 69.

[82] A. Frommer, K. Kahl, S. Krieg, B. Leder, and M. Rottmann, Adaptive aggregation based domain decomposition multigrid for the lattice Wilson Dirac operator, SIAM J. Sci. Comput. 36, A1581 (2014). 